\title{
Model depiction of the atmospheric flows of radioactive cesium emitted from the Fukushima Daiichi Nuclear Power Station accident
}

\author{
Teruyuki Nakajima ${ }^{1,2^{*}}$, Shota Misawa ${ }^{1}$, Yu Morino ${ }^{3}$, Haruo Tsuruta ${ }^{1,4}$, Daisuke Goto ${ }^{3}$, Junya Uchida ${ }^{1}$, \\ Toshihiko Takemura ${ }^{5}$, Toshimasa Ohara ${ }^{3}$, Yasuji Oura ${ }^{6}$, Mitsuru Ebihara ${ }^{6}$ and Masaki Satoh ${ }^{1}$
}

\begin{abstract}
In this study, a new method is proposed for the depiction of the atmospheric transportation of the ${ }^{137} \mathrm{Cs}$ emitted from the Fukushima Daiichi Nuclear Power Station accident. This method employs a combination of the results of two aerosol model ensembles and the hourly observed atmospheric ${ }^{137} \mathrm{Cs}$ concentration at surface level during $14-$ 23 March 2011 at 90 sites in the suspended particulate matter monitoring network. The new method elucidates accurate transport routes and the distribution of the surface-level atmospheric ${ }^{137} \mathrm{Cs}$ relevant to eight plume events that were previously identified. The model ensemble simulates the main features of the observed distribution of surface-level atmospheric ${ }^{137} \mathrm{Cs}$. However, significant differences were found in some cases, and this suggests the need to improve the modeling of the emission scenario, plume height, wet deposition process, and plume propagation in the Abukuma Mountain region. The contributions of these error sources differ in the early and dissipating phases of each event, depending on the meteorological conditions.
\end{abstract}

Keywords: Fukushima Nuclear Power Station accident, Aerosols, Radioactive materials, ${ }^{137} \mathrm{Cs}$, Chemical transport modeling, Ensemble models

\section{Introduction}

A wide area of northeastern Japan, the Tohoku and Kantou regions, was contaminated by the radioactive material emitted from the accident at the Fukushima Daiichi Nuclear Power Station (FDNPS) of the Tokyo Electric Power Company (TEPCO), as manifested by various environmental investigations (Nakajima et al. 2014). The accident was caused by the Great East Japan Earthquake, which struck at 14:46 Japan Standard Time (JST; Coordinated Universal Time, UTC+ 9 h) on 11 March 2011.

Takemura et al. (2011) show that the negative anomaly of a 500-hPa height over the Okhotsk Sea area along $145^{\circ}$ $E$ made the westerly jet stronger than the climatological

\footnotetext{
* Correspondence: terry-nkj@nifty.com

${ }^{1}$ Atmosphere and Ocean Research Institute (AORI), The University of Tokyo, 5-1-5 Kashiwanoha, Kashiwa, Chiba 277-8568, Japan

${ }^{2}$ Earth Observation Research Center (EORC), Japan Aerospace Exploration

Agency (JAXA), 2-1-1 Sengen, Tsukuba, Ibaraki 305-8505, Japan

Full list of author information is available at the end of the article
}

mean during mid-March; consequently, 70 to $80 \%$ of the radioactive material from the FDNPS was driven to the Pacific Ocean and the rest of the globe (Takemura et al. 2011; Stohl et al. 2012; Mészáros et al. 2016). The remaining material spread over and deposited onto the land area of Japan, producing characteristic hot spot patterns (Yasunari et al. 2011; JAEA 2012; SCJ 2014). The total emission of ${ }^{137} \mathrm{Cs}$ into the atmosphere until the end of April was estimated to be $14.6 \pm 3.5 \mathrm{PBq}$ (SCJ 2014). The ratio of the total deposition over the Japanese land area to the total atmospheric emission was estimated as $20 \pm 6 \%$, according to the airborne monitoring conducted by the Ministry of Education, Culture, Sports, Science, and Technology, Japan (MEXT 2011), whereas the ratio was calculated as $27 \pm 10 \%$ based on the multi-model intercomparison by the Science Council of Japan (SCJ 2014). To date, this inconsistency has not been fully understood, owing to the lack of observation data, which is attributable to instrumental damage and electric outages 
as well as modeling uncertainties. In addition, there is still great uncertainty in the emission time series of the radioactive material, as shown in Fig. 1. Yumimoto et al. (2016) conducted an inverse analysis to optimally estimate the emission rate using the time series of the deposition map, but the result is very different from that of Katata et al. (2015).

Recently, Tsuruta et al. (2014) developed a method to directly measure the hourly time series of the atmospheric ${ }^{137} \mathrm{Cs}$ concentration at surface level, from the aerosol sampling tapes of the national suspended particulate matter (SPM) network. The SPM network monitors air pollution by employing beta-ray attenuation counters. Four laboratories, namely, those of Tokyo Metropolitan University, the Nuclear Professional School of the University of Tokyo, the Japan Atomic Energy Agency, and the Japan Chemical Analysis Center, retrieved the atmospheric loading from the hourly aerosol spots on the SPM tape. This method offers the potential for studying the atmospheric transport of ${ }^{137} \mathrm{Cs}$, although the data is from surface level, during the entire post-accident period; the SPM dataset has high temporal and spatial sampling, with observations every hour at 90 out of 400 sites (Fig. 2). In Fig. 2, it can be seen that the Nakadori region is a channel basin area between the $\mathrm{Ou}$ and Abukuma mountains, while the Hamadori region is a coastal region to the east of the Abukuma mountains. The FDNPS is located in the northern part of the Hamadori region. In this report, we compare the ensemble results of two aerosol transport models with SPM data. An important purpose of the comparison is to investigate the validity of the combined use of SPM data and multi-model simulations to depict the transportation of atmospheric ${ }^{137} \mathrm{Cs}$ over the Japan land area. Once validated, further analysis can be performed on a larger volume of SPM data, such as the most recent data from 99 SPM sites, which has recently been made available to the public (Oura et al. 2015). In addition, the results could be a useful input for our second model intercomparison, which is intended as a follow-up to the first comparison, which was made by the SCJ (SCJ 2014), and this can contribute to future discussions of the use of models in emergency protocols.

Tsuruta et al. (2014) identified nine plumes, as listed in Fig. 3, that transported particulates to the land area of
Japan and in which the maximum atmospheric ${ }^{137} \mathrm{Cs}$ concentration exceeded $10 \mathrm{~Bq} \mathrm{~m}{ }^{-3}$, based on a synoptic analysis using a time series of the SPM data and the wind vector field. For purposes of comparison, we selected plumes P2 to P9 in the period 14-24 March 2011. In this period, there were two migrations of low pressure systems over Japan; these occurred on 15 and 20 March, according to the weather maps shown in Fig. 4.

\section{Methods/Experimental \\ Model simulation of the atmospheric ${ }^{137} \mathrm{Cs}$ concentration at surface level}

In this study, two aerosol transport models were employed to simulate the atmospheric transportation of ${ }^{137} \mathrm{Cs}$. The first model uses an online dynamic core of the nonhydrostatic icosahedron atmospheric model (NICAM; Tomita and Satoh 2004; Satoh et al. 2008) coupled with the spectral radiation-transport model for aerosol species (SPRINTARS; Takemura et al. 2000; Dai et al. 2014); we will refer to this as the N-model. The NICAM model is implemented with an air-mass flux-type dynamic scheme of high mass conservation, and it can cover a global-to-regional simulation with its three grid systems, which include a quasihomogeneous global grid, a stretched grid, and a diamond regional grid (Uchida et al. 2015). We adopted the diamond grid system for a regional simulation with the following settings: $10 \mathrm{~s}$ time step, $5-\mathrm{km}$ grid resolution, and 40 layers, with the lowest layer being $20 \mathrm{~m}$ thick. The cloud microphysics scheme was the NICAM singlemoment scheme with six water categories (NSW6), which is a simplified version of Lin's scheme (Lin et al. 1983). The turbulent diffusion calculation adopted a partial condensation scheme (Mellor and Yamada 1982) and an eddy diffusion scheme with a level 2.0 turbulent scale adjustment scheme (Nakanishi and Niino 2004). The numerical domain was a rhombus area, with apexes at $26.5^{\circ} \mathrm{N}, 144.4^{\circ} \mathrm{E}$; $35.8^{\circ} \mathrm{N}, 132.0^{\circ} \mathrm{E} ; 50.6^{\circ} \mathrm{N}, 133.4^{\circ} \mathrm{E}$; and $39.9^{\circ} \mathrm{N}, 147.7^{\circ} \mathrm{E}$; it covered the Tohoku region (the northeastern part of the Japanese islands, including the Fukushima and Miyagi prefectures, as shown in Fig. 2) and the Kantou Plain region (the area including the Tokyo, Saitama, Chiba, Kanagawa, and Ibaraki prefectures). After release from the FDNPS, the atmospheric ${ }^{137} \mathrm{Cs}$ was treated as a sulfate aerosol particle

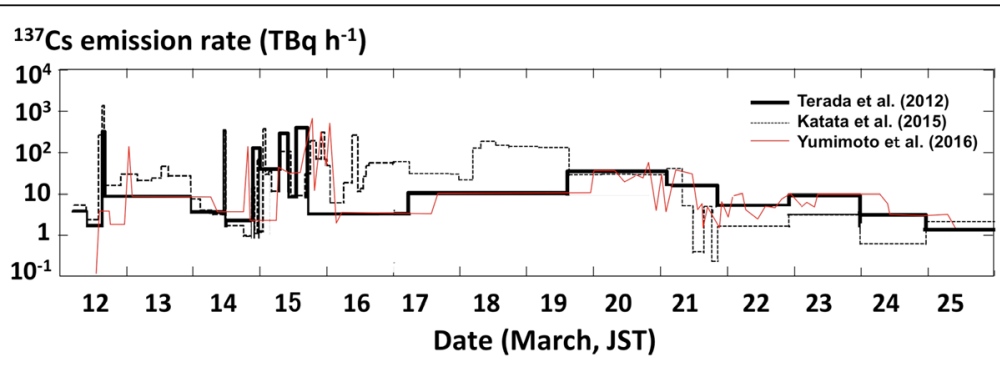

Fig. 1 Time series of the ${ }^{137} \mathrm{Cs}$ emission rate from the FDNPS, as estimated by Terada et al. (2012), Katata et al. (2015), and Yumimoto et al. (2016) 


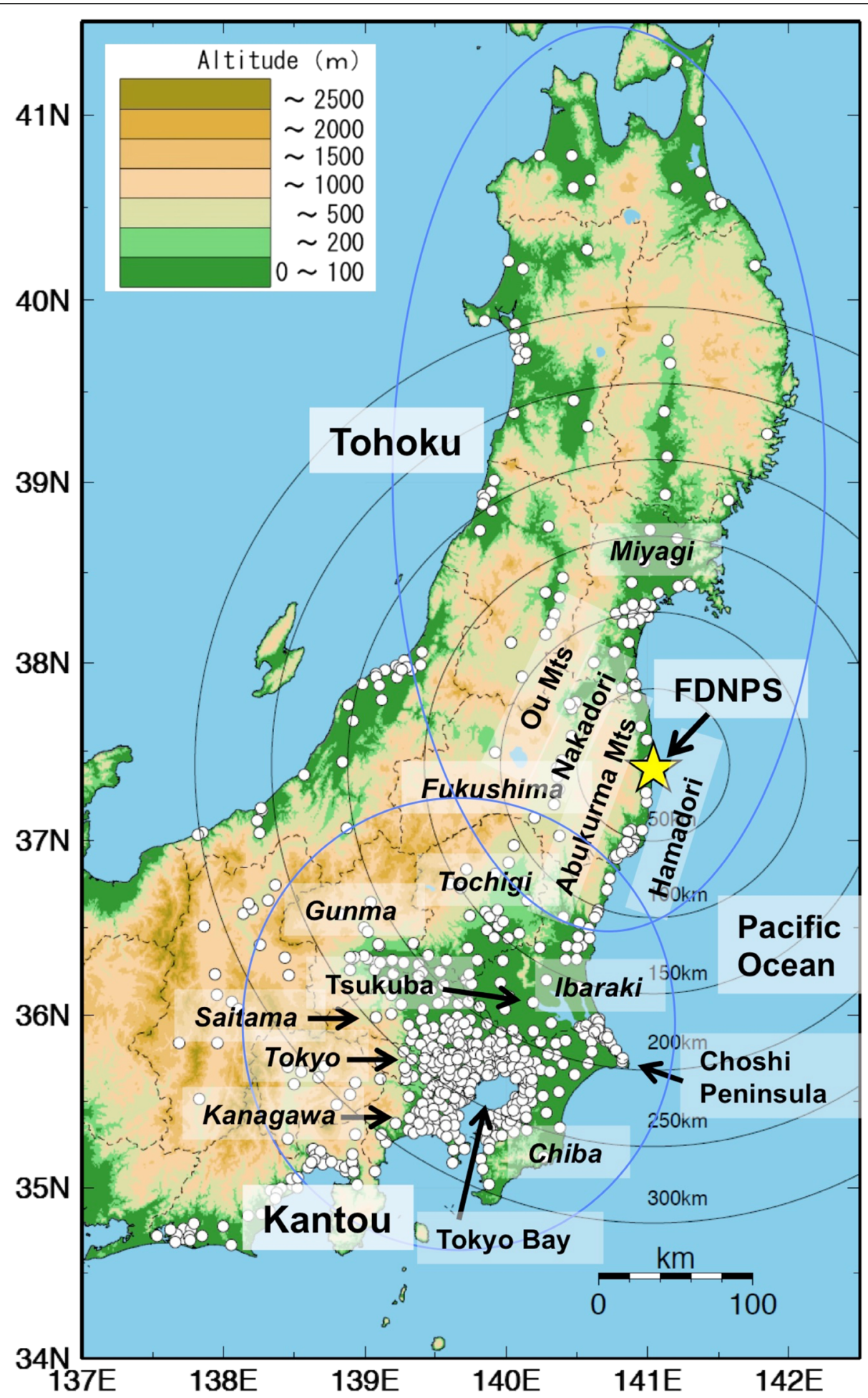

Fig. 2 Names of key regions and locations of SPM sites at the time of accident for the present study. The Tohoku region is the northeastern part of the Japanese islands and includes the Fukushima and Miyagi prefectures; the Kantou region is the area that includes the Tokyo, Saitama, Chiba, Kanagawa, and Ibaraki prefectures. The FDNPS is located in the northern part of the Hamadori region, a coastal area to the east of the Abukuma Mountains. The Nakadori region is a channel basin area between the Ou and the Abukuma mountains. Open circles are SPM monitoring sites managed and maintained by local governments in eastern Japan before the accident. The base map was modified by using the original map in Fig. 1 of Tsuruta et al. (2014) 


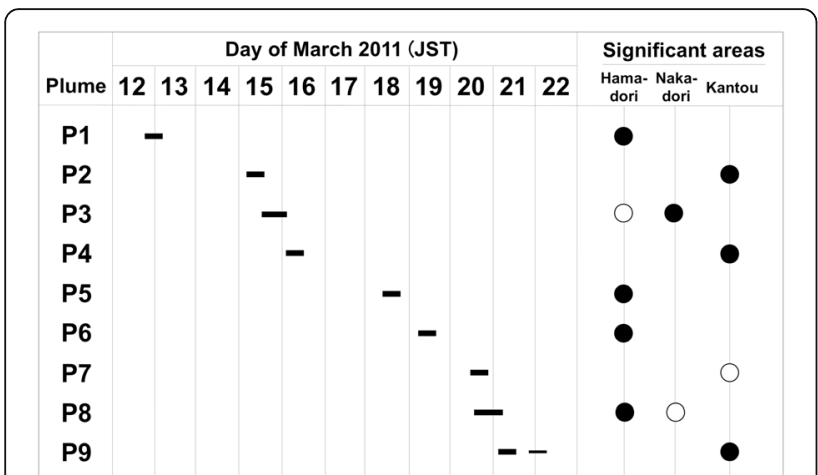

Fig. 3 Plumes identified by Tsuruta et al. (2014). Horizontal bars show the period with high ${ }^{137} \mathrm{Cs}$ concentrations $\left(>10 \mathrm{~Bq} \mathrm{~m}^{-3}\right)$. Closed and open circles indicate areas in the Hamadori, Nakadori, and Kantou regions where the concentrations were larger or smaller, respectively, than $100 \mathrm{~Bq} \mathrm{~m}^{-2}$

and assumed to undergo transportation, hygroscopic growth, dry and wet deposition, and gravitational settling, according to the scheme of Takemura et al. (2000). The present numerical experiment assumed a monodisperse sulfate-equivalent particle with a radius of
$0.24 \mu \mathrm{m}$, taking into account the observed size distribution by Kaneyasu et al. (2012) and an in-cloud scavenging coefficient defined by Takemura et al. (2000) as 0.8 (Goto et al. 2015a).

The other model was a meteorological model, the weather research and forecast model (WRF) version 3.1 (Skamarock et al. 2008), coupled with a three-dimensional chemical transport model, the Models-3 Community Multiscale Air Quality (CMAQ) version 4.6 (Byun and Schere 2006); we will refer to this as the W-model. The CMAQ had been modified for radioactive material transport by the National Institute for Environmental Studies for a FDNPS simulation (Morino et al. 2011; SCJ 2014). We employed the WRF modules of scalar positive-definite advection, full diffusion, without the sixth-order horizontal-advection noise filter, and the planetary boundary parameterization of the Mellor-Yamada-Janjic scheme (Wang et al. 2012). In the present simulation, all the ${ }^{137} \mathrm{Cs}$ was assumed to be in particulate phase (Sportisse 2007), and the chemical and aerosol processes were not calculated because no detailed process has been well reported. The deposition schemes were those of Byun and Ching (1999) and Byun and Schere (2006). The mean particle radius was assumed to
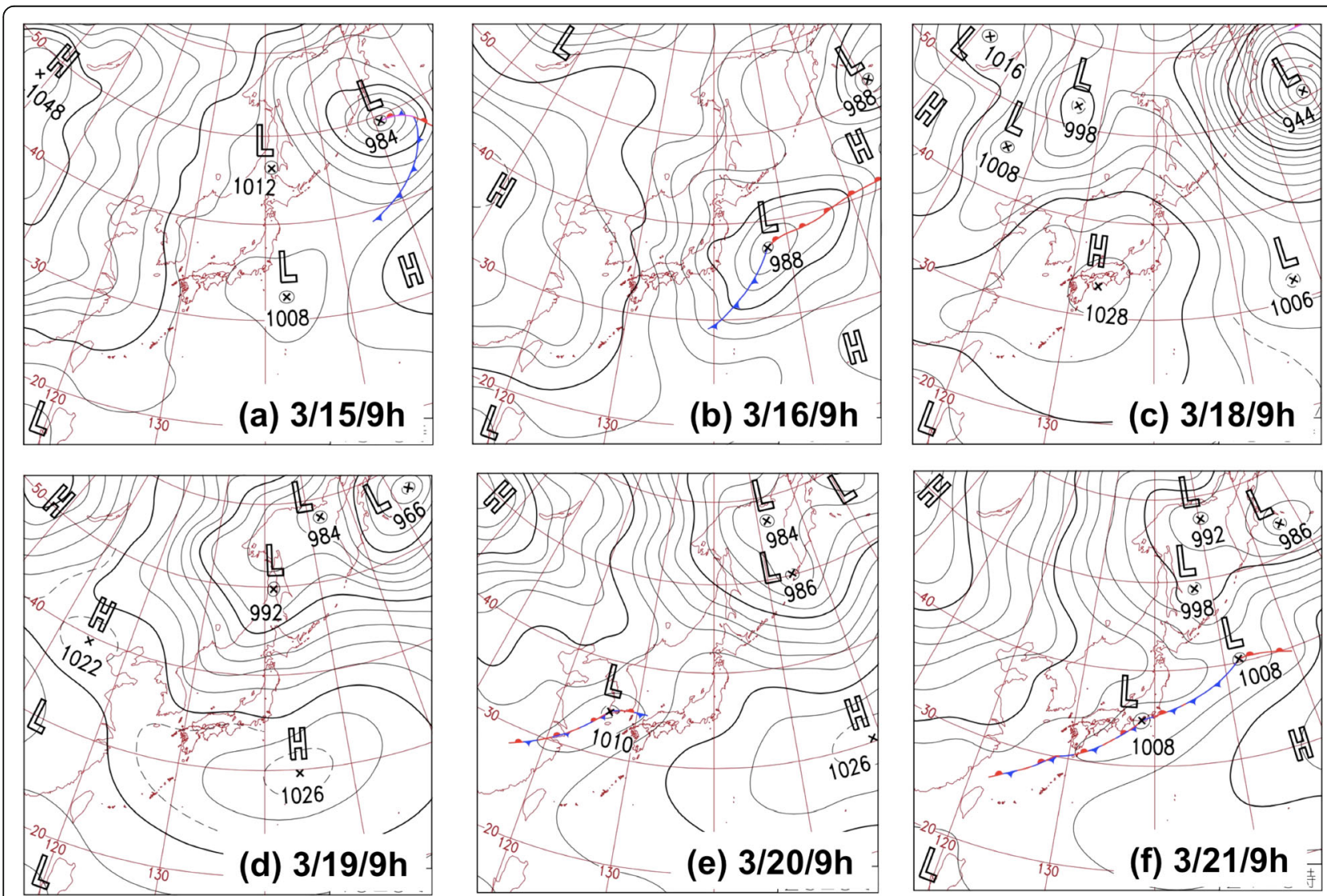

Fig. 4 a-f Weather maps based on JMA analysis at 9:00 JST in the analysis period 
be $0.5 \mu \mathrm{m}$, and the geometrical dispersion $\sigma_{\mathrm{g}}$ was assumed to be 1.1 (Sparmacher et al. 1993; Sportisse 2007; Kaneyasu et al. 2012). The model domain was a $700 \times 700 \mathrm{~km}^{2}$ area, covering most of the Tohoku and Kantou regions, with a grid resolution of $3 \mathrm{~km}$ and 34 layers, with the lowest layer approximately $60 \mathrm{~m}$ thick. The simulation period was 14-24 March, and the analysis was performed by comparing the model ensemble results with the observed atmospheric ${ }^{137} \mathrm{Cs}$ concentration at surface level. The total deposition over Japanese land was simulated as $2.12 \mathrm{PBq}$ by the Nmodel and $2.21 \mathrm{PBq}$ by the $\mathrm{W}$-model (Morino et al. 2013). These values belong to the small deposition group in the SCJ model comparison (SCJ 2014), which gave a mean value of total deposition of $2.92 \mathrm{PBq}$.

Both models were nudged with three-hourly $5 \times 5 \mathrm{~km}$ mesoscale objective analysis data (MANAL) from the Japan Meteorological Agency (JMA). We selected the emission scenario of Terada et al. (2012), which has been used in many previous studies and is thus suitable for comparison with them. Figure 5 shows two examples of the simulated and observed atmospheric ${ }^{137} \mathrm{Cs}$ used to study the performance of both the $\mathrm{N}$ - and the $\mathrm{W}$-model for specific cases, taken from the detailed time sequences as shown in Figs. 6 and 7 with precipitation maps of the JMA analysis and the $\mathrm{N}$-model. These two examples were $15 \mathrm{March}, 12: 00 \mathrm{JST}$ (3/15/12 h), and 21 March, 9:00 JST (3/21/9 h), when highconcentration plumes of more than $100 \mathrm{~Bq} \mathrm{~m}^{-3}$ were observed in the Kantou region. In Fig. 5, observed concentrations are indicated by color-coded circles, and the data below the detection limit of $0.1 \mathrm{~Bq} \mathrm{~m}^{-3}$ are indicated by triangles. On 15 March, a dry process governed the transportation, and there was no significant precipitation until late that evening, as indicated by the JMA analysis shown in Fig. 6. The distribution of the atmospheric ${ }^{137} \mathrm{Cs}$ was well simulated by the two models for a wide range of concentrations, from 0.1 to $100 \mathrm{~Bq} \mathrm{~m}{ }^{-3}$. It should be noted that the observed high-concentration area was confined to a narrow region, which was consistent with the simulated plume-wise distribution starting from the FDNPS for the Kantou region. The plumes simulated by the $\mathrm{N}$-model were less diffused (narrower) than those simulated by the W-model. Judging by the correlation coefficients of the observed and simulated distributions, reality is assumed to fall between these two results; this is further discussed in the following paragraph.

For the March 21 event, the results of the N- and Wmodels differed significantly, as can be seen in Fig. $5 c$, d, respectively. Morning precipitation occurred in the Kantou region on 21 March, as will be shown in Fig. 7b. However, the simulated atmospheric concentration of ${ }^{137} \mathrm{Cs}$ was generally smaller than the observed values, suggesting that the simulated wet deposition was too strong for the W-model. Wet deposition during the total period over land in Japan was simulated as 1.68 and $2.12 \mathrm{PBq}$ by the $\mathrm{N}$ - and W-model, respectively.
As indicated in Fig. 5, the accuracies of the simulated plume patterns of the two models differed from event to event. These differences were also reported by SCJ (2014). One way to reconcile the differences and construct statistically optimum distributions is to use a multi-model ensemble method to calculate the weighted mean concentration $\bar{C}_{k}$ for the $k$ th grid point of the $\mathrm{N}$-model at the $j$ th time step, as given by

$$
\bar{C}_{k, j}=\left(\frac{C_{\mathrm{N}, k, j}}{\sigma_{\mathrm{N}}^{2}}+\frac{C_{\mathrm{W}, k, j}}{\sigma_{\mathrm{W}}^{2}}\right) /\left(\frac{1}{\sigma_{\mathrm{N}}^{2}}+\frac{1}{\sigma_{\mathrm{W}}^{2}}\right),
$$

where $C_{N, k, j}$ and $C_{W, k, j}$ are the values of the $\mathrm{N}$ - and Wmodel, respectively. The grid values of the $\mathrm{W}$-model are linearly interpolated onto the $\mathrm{N}$-model grid. The quantities $\sigma_{\mathrm{N}}$ and $\sigma_{\mathrm{W}}$ are the daily root mean square deviations of the respective model values from the observed values. Figure 8 shows a comparison of the observed values with the values obtained by the $\mathrm{N}$-model alone and with the two-model ensemble means constructed by the proposed method. The daily correlation coefficients for specific days $(15,20$, and 21 March) are listed in Table 1. The correlation coefficients were calculated using the daily means of the observed and simulated values at the surface level, as linearly interpolated at the site locations. The ensemble method constructs an atmospheric ${ }^{137} \mathrm{Cs}$ distribution that had a correlation coefficient better than that of either model alone. However, 20 March was an exception; the correlation coefficient of the two-model ensemble method was not as good as that of the N-model, although it was superior to that of the W-model. The 3-day average of the correlation coefficients was improved to 0.51 by the two-model ensemble method, from 0.48 obtained with the $\mathrm{N}$-model and 0.46 with the $\mathrm{W}$-model.

\section{Results and discussion \\ Structure of atmospheric ${ }^{137} \mathrm{Cs}$ plumes}

In this section, we examine the plume formation and dissipation during each of three periods: 15-16 March, 18-19 March, and 20-21 March 2011.

\section{5-16 March}

Land plume events in the Kantou and Tohoku regions lasted from the morning of 15 March until noon of the next day. Time sequences of the ${ }^{137} \mathrm{Cs}$ concentration of the two models and the observations are shown in Fig. $6 a, b$, along with precipitation maps of the JMA analysis and those of the N-model. Two-model ensemble means and the wind vectors of the JMA analysis are shown in Fig. 9 for several characteristic times. During the morning of 15 March, a low-pressure system with a weak trough structure was located in the Pacific region off the Kantou region (Fig. 4a). A northeasterly wind 


\section{$3 / 15 / 12 h$}
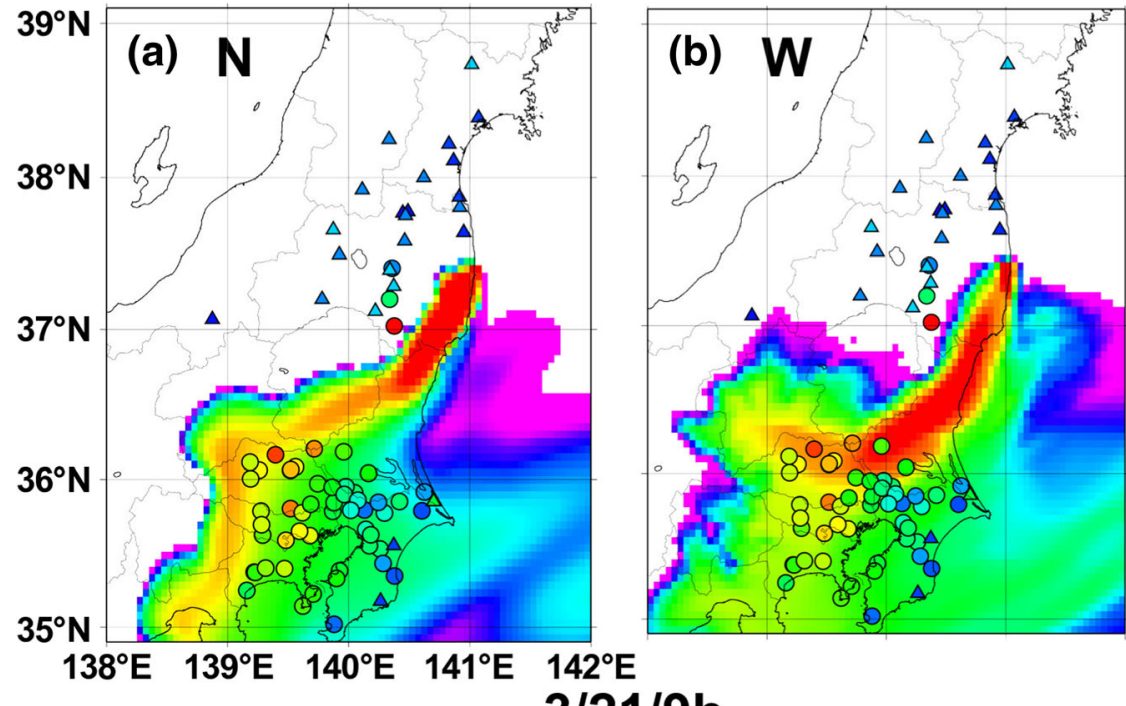

$3 / 21 / 9 h$
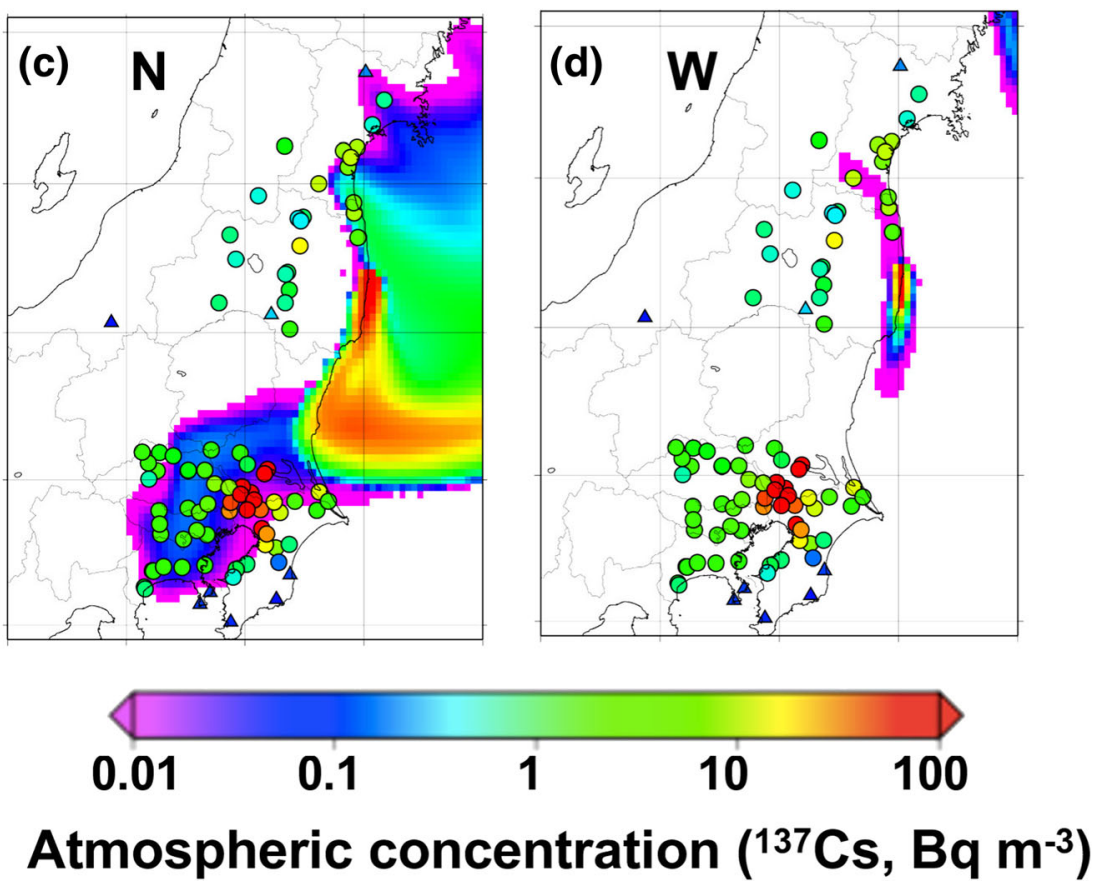

Fig. 5 Comparison of the atmospheric ${ }^{137} \mathrm{Cs}\left(\mathrm{Bq} \mathrm{m}^{-3}\right)$ for events on 15 March, 12:00 and 21 March, 9:00, as estimated by the NICAM-SPRINTARS model $(N ; \mathbf{a}, \mathbf{c})$ and the WRF-CMAQ model $(W ; \mathbf{b}, \mathbf{d})$. Observed values are indicated by color-coded circles $\left(B q \mathrm{~m}^{-3}\right)$; triangles indicate data below the detection limit $\left(0.1 \mathrm{~Bq} \mathrm{~m}^{-3}\right)$ of SPM measurements

prevailed at the northern edge of the low-pressure system until 9:00, replacing the southwesterly wind associated with the high-pressure system of the previous day (not shown). This northeasterly wind transported radioactive material from the FDNPS southward to form plume P2, departing from the FDNPS at midnight and arriving at the Kantou region around 4:00 (Fig. 6a, panel 4:00), at which point the concentration exceeded several $\mathrm{Bq} \mathrm{m}{ }^{-3}$, according to observations by the SPM network. Figure 6a, panels 0:00 to 4:00, indicates that the arrival time and location of the tongue of simulated plume P2 appear to be consistent with the observed concentrations shown by color-coded circles. The model simulation of plume transportation was relatively easy in this early phase during which there was no precipitation. 
(a)
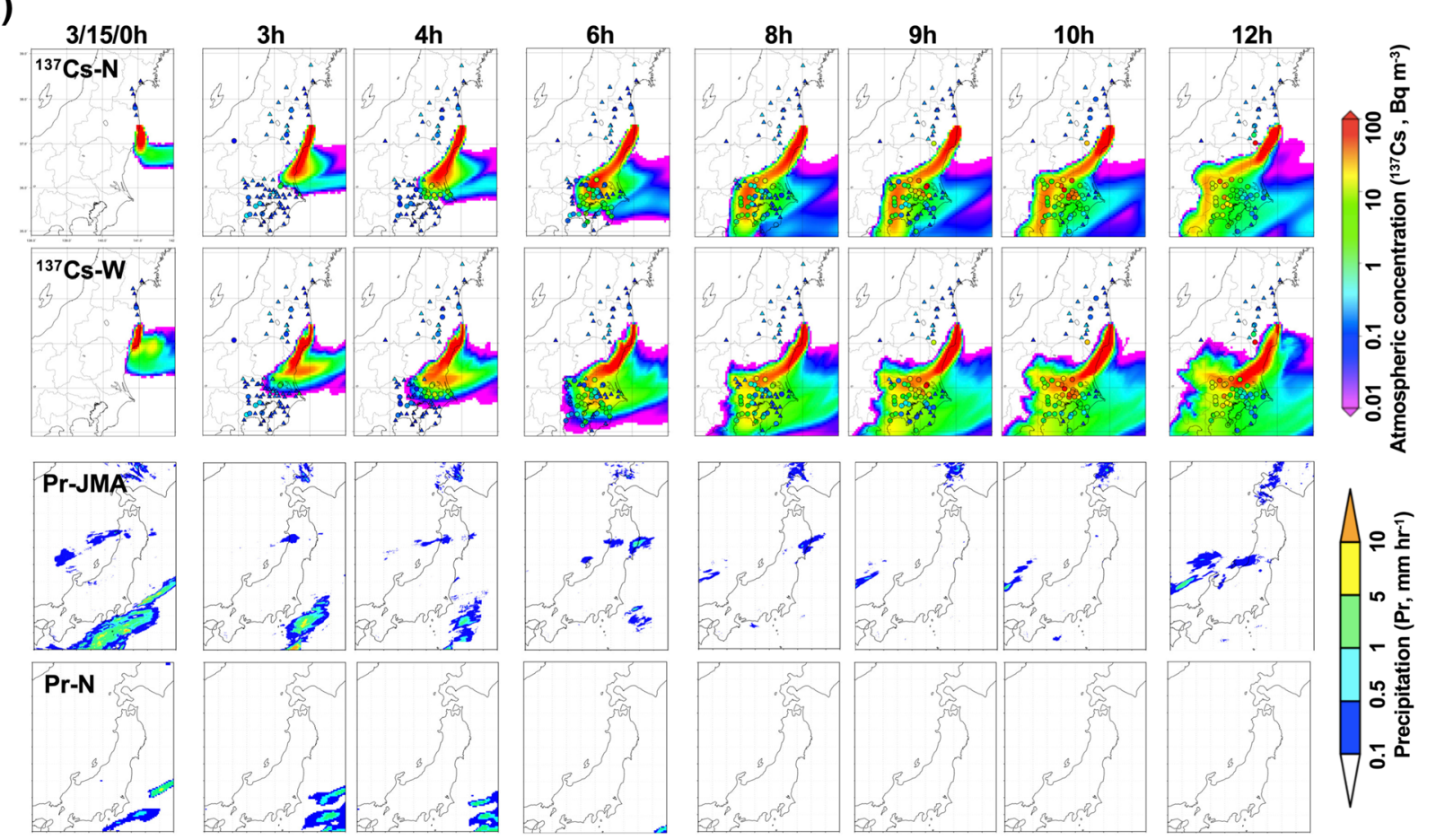

(b) $3 / 15 / 15 \mathrm{~h}$
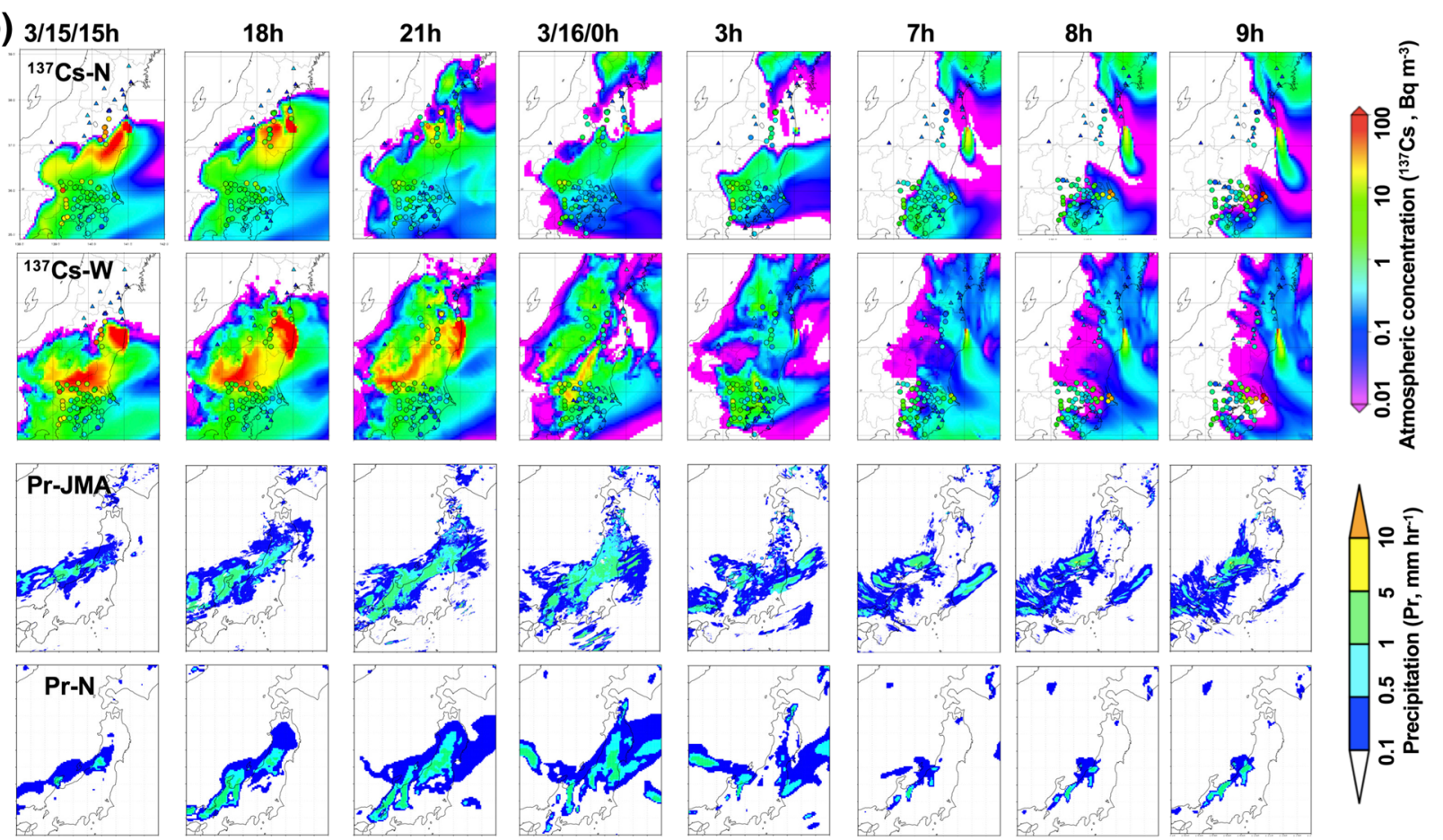

Fig. 6 Maps of the atmospheric ${ }^{137} \mathrm{Cs}$ concentration at surface level as simulated by the $\mathrm{N}$ - and $\mathrm{W}$-models for the periods of a 3/15/0 $\mathrm{h}$ to $3 / 15 /$ $12 \mathrm{~h}$ and $\mathbf{b}$ 3/15/15 $\mathrm{h}$ to 3/16/9 h. The observed concentrations are indicated by colored circles. Precipitation maps are also shown for the JMA analysis and the $\mathrm{N}$-model

Simulation results of the maximum stage of plume P2 in the Kantou region is, however, different from the SPM observations; a thick simulated plume as large as tens of $\mathrm{Bq} \mathrm{m} \mathrm{m}^{-3}$ arrived around 6:00 at the Saitama, Tokyo, and Kanagawa prefectures (Fig. 6a, panels 6:00 to 9:00, and Fig. 9a, b), whereas the observed data did not 


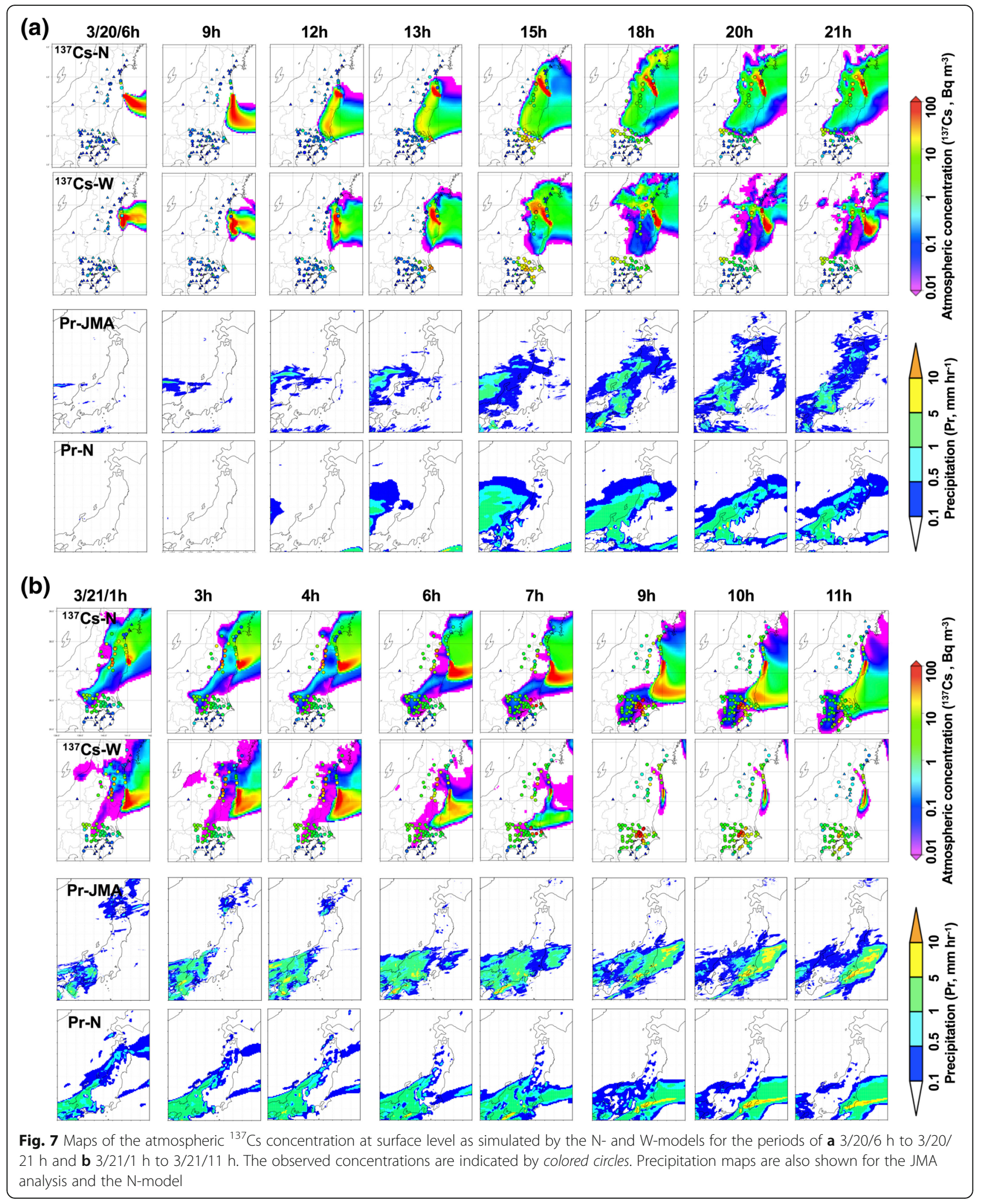

show an increase to more than $10 \mathrm{~Bq} \mathrm{~m}^{-3}$ until 9:00, indicating a later arrival. Such a large difference in the arrival time of the plume cannot be explained by model errors, given that there were relatively simple meteorological conditions with a dry process, and the $\mathrm{N}$ - and W-models had similar results, as shown in Fig. 6a, panels 0:00 to 9:00. 


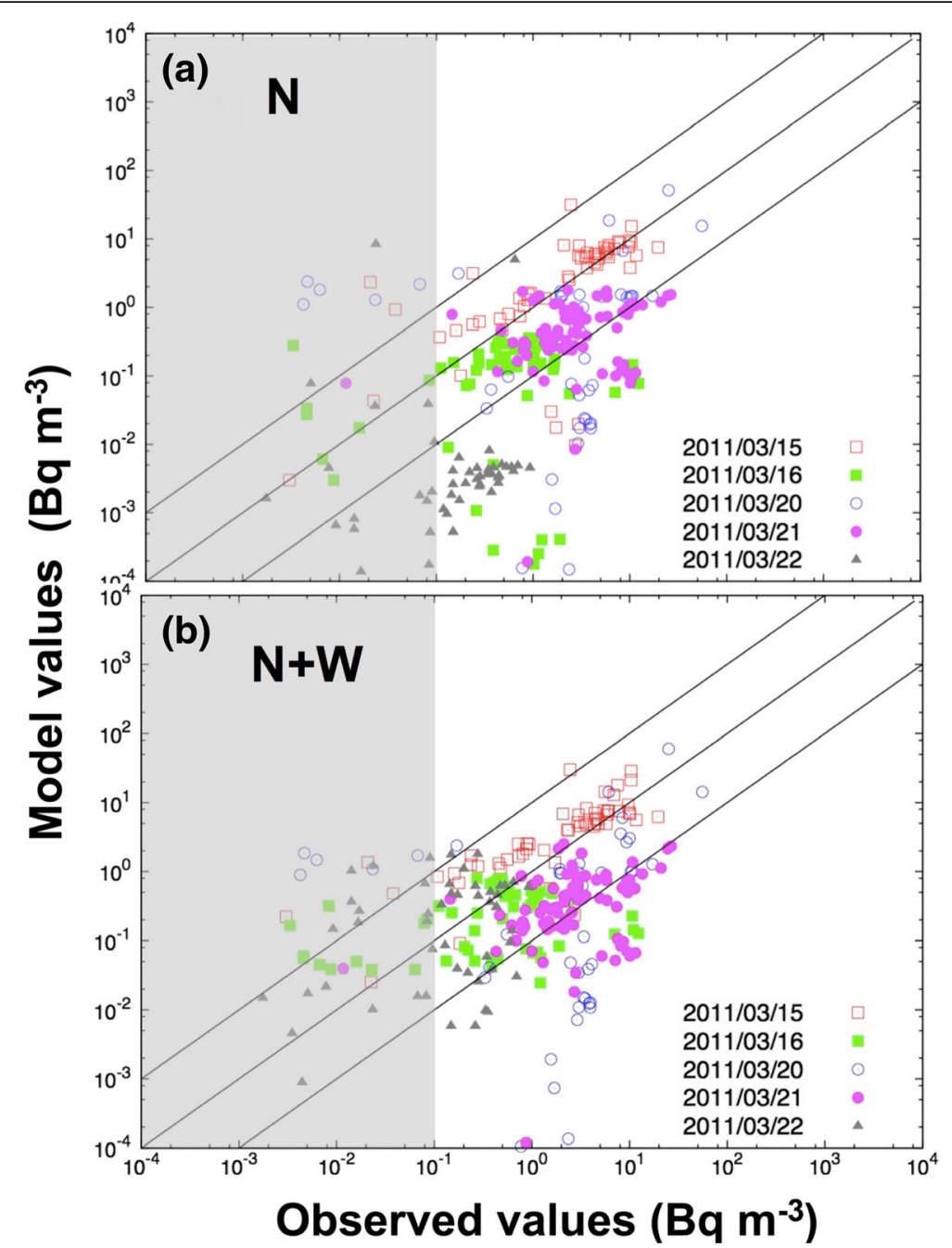

Fig. 8 Scatter plots of the observed atmospheric ${ }^{137} \mathrm{Cs}$ concentration $\left(\mathrm{Bq} \mathrm{m}^{-3}\right)$ and the values obtained by the $\mathrm{N}$-model and the two-model ensemble mean $(\mathbf{a}, \mathbf{b})$. The area in which the data was below the detection limit $\left(0.1 \mathrm{~Bq} \mathrm{~m}^{-3}\right)$ is shaded

One possible cause of this is the large emission from FDNSP around 22:00 on 14 March, in the emission scenario of Terada et al. (2012). Figure 1 shows that the updated scenario of Katata et al. (2015), on the other hand, suggests a large emission occurred later, around 1:00 on 15 March. To investigate this possibility, Fig. 10 shows the simulation results of the $\mathrm{N}$-model for the scenario of Terada et al. (2012) and that of Katata et al.

Table 1 Daily correlation coefficients between observed and simulated ${ }^{137} \mathrm{Cs}$ concentrations based on N-model, W-model, and two-model ensemble $(\mathrm{N}+\mathrm{W})$ results for ${ }^{137} \mathrm{Cs}$

\begin{tabular}{llll}
\hline Day (JST) & N & W & N +W \\
\hline $2011 / 3 / 15$ & 0.51 & 0.52 & 0.55 \\
$2011 / 3 / 20$ & 0.57 & 0.50 & 0.55 \\
$2011 / 3 / 21$ & 0.36 & 0.37 & 0.44 \\
Average & 0.48 & 0.46 & 0.51 \\
\hline
\end{tabular}

(2015). It can be seen in the figure that with the scenario of Katata et al. (2015), the high-concentration area in the Kantou region, becomes smaller and is more consistent with the observations.

The observed high-concentration area of plume P2 gradually spread from 9:00 to 12:00 in the Central Tokyo area, which is similar to simulations that show that the plume slightly shifted to the east, as shown in Fig. 6a, panels 9:00 to 12:00. Figure 9c shows that the ensemble model cannot account for this shift of the highconcentration area, but the emission scenario of Katata et al. (2015) gives a better result (Fig. 10). The SPM data suggest that dissipation of the P3 plume occurred during the evening of 15 March through the morning of 16 March, as shown in Fig. 6, panels 3/15/15:00 to 3/16/ 7:00, and Fig. 9d, e. The $\mathrm{N}$-model adequately simulated the timing and distribution of the plume dissipation following a reduction in the transport of radioactive 


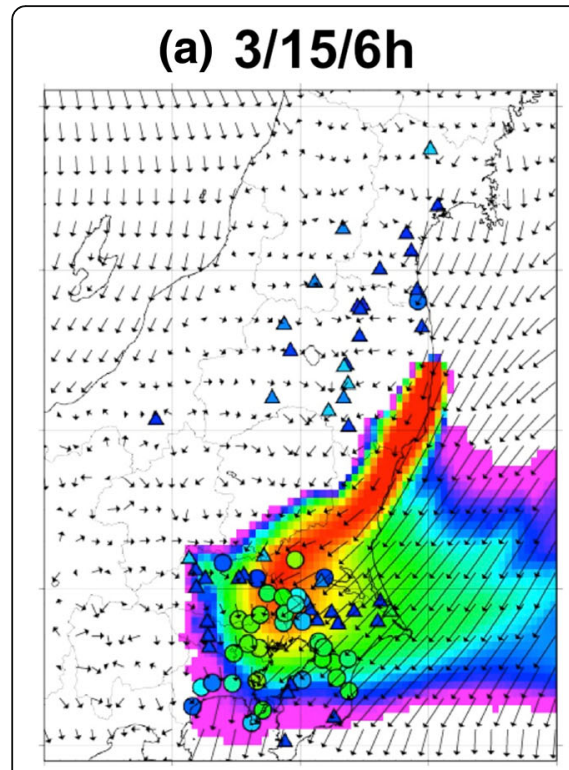

(d) $3 / 15 / 15 \mathrm{~h}$
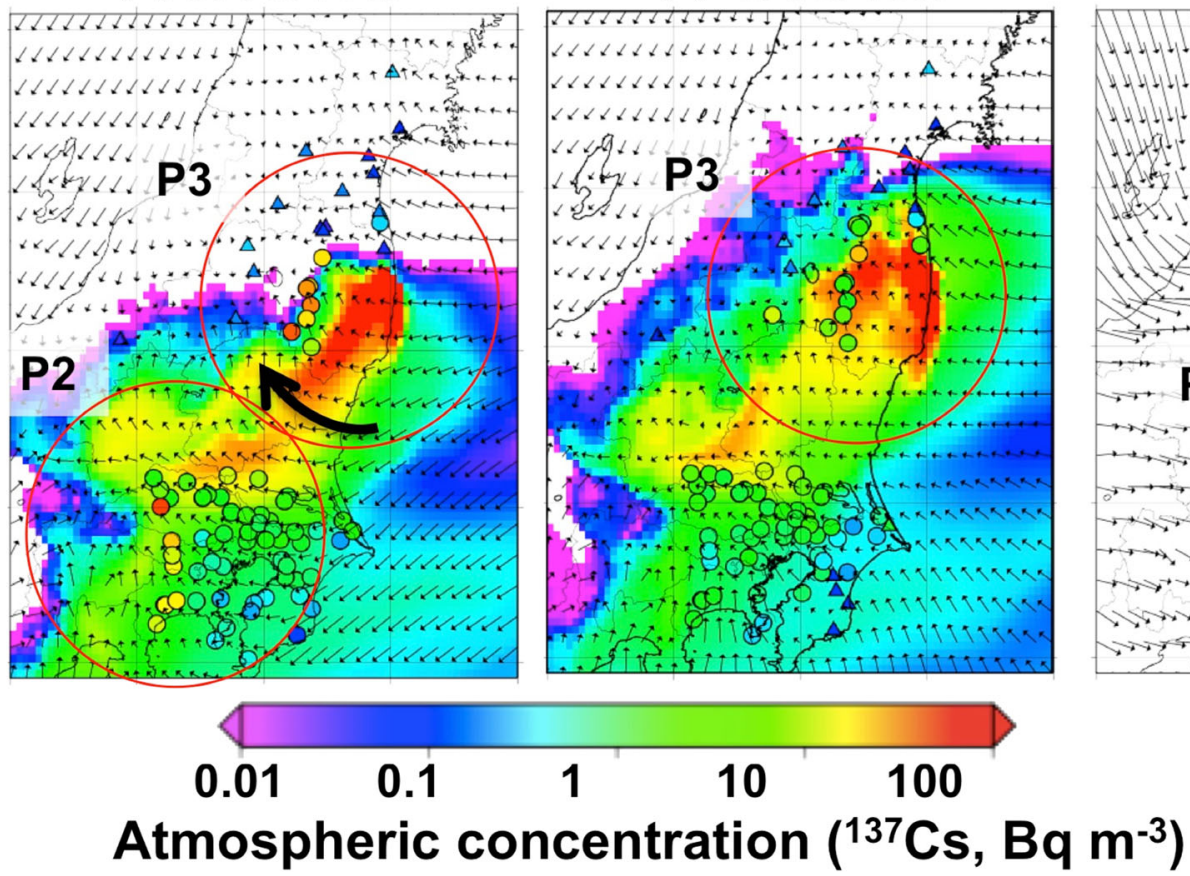

(b) $3 / 15 / 9 \mathrm{~h}$

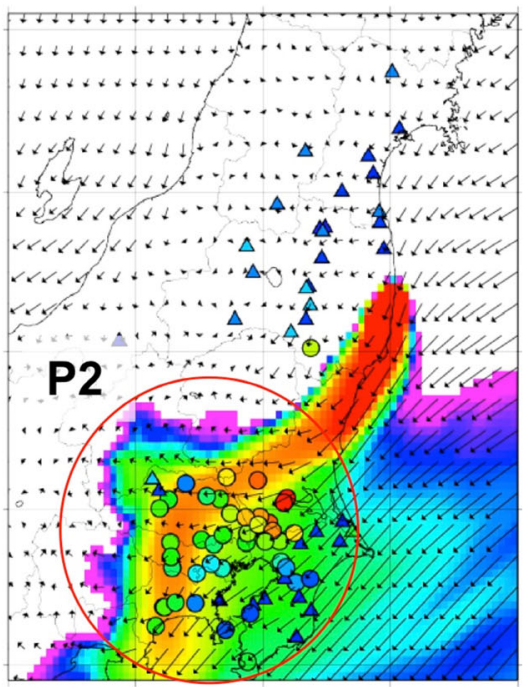

(e) $3 / 15 / 18 \mathrm{~h}$

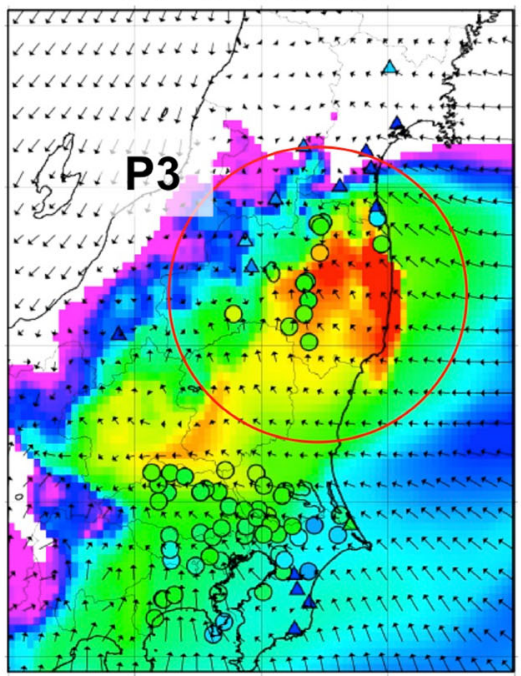

Cs concentration from $3 / 15 / 9 \mathrm{~h}$ to $3 / 16 / 9 \mathrm{~h}$. Areas

Fig. 9 a-f Maps of observations (colored circles) and model ensemble means of atmospheric ${ }^{137} \mathrm{Cs}$ concentration from $3 / 15 / 9 \mathrm{~h}$ to $3 / 16 / 9 \mathrm{~h}$. Areas
identified as plumes with concentration larger than $10 \mathrm{~Bq} \mathrm{~m} \mathrm{~m}^{-3}$ are encircled and labeled with the name of the plume. JMA-MANAL wind vectors at $1000 \mathrm{hPa}$ are superimposed. Movement of the P3 plume route is indicated by a thick arrow in panel $\mathbf{d}$ (c) $3 / 15 / 12 \mathrm{~h}$

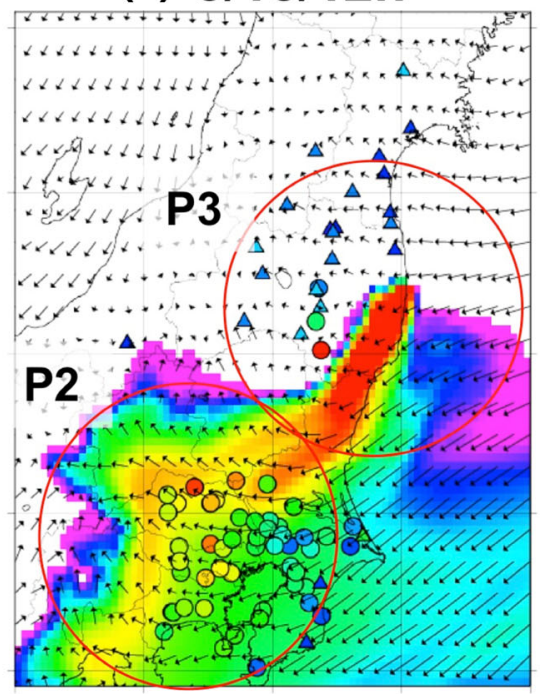

(f) $3 / 16 / 9 \mathrm{~h}$

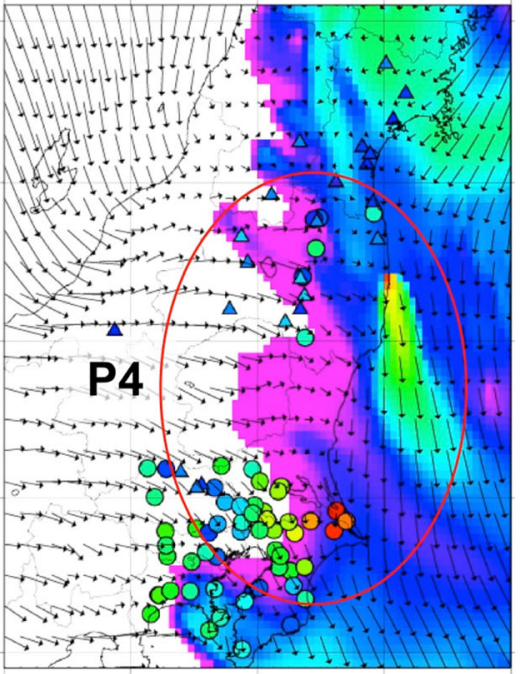

$\leftarrow 10 \mathrm{~ms}^{-1}$ material, due to a patch of precipitation between FDNSP and Kantou; however, the W-model simulation showed a slower dissipation.

The wind vector fields shown in Fig. 9c, d indicate that a weak cyclonic motion around the Central Fukushima area was generated until noon of 15 March; this was probably due to solar heating. It cause a gradual spread of plume P2 toward the western part of the Tokyo area, as indicated by a thick arrow in Fig. 9d. The wind field also transported plume P3 toward the Nakadori region in the afternoon of $15 \mathrm{March}$, when high concentrations of more than $100 \mathrm{~Bq} \mathrm{~m}^{-3}$ were observed consecutively from southern to northern sites (Fig. 6, panels 12:00 to 15:00, and Fig. 9c, d). In the late afternoon, a southeasterly wind became dominant at the FDNSP, and consequently, a part of the P3 plume took a northern detour to the edge of the Abukuma mountains and into the Nakadori region from north to south, as shown by 


\section{Terada}
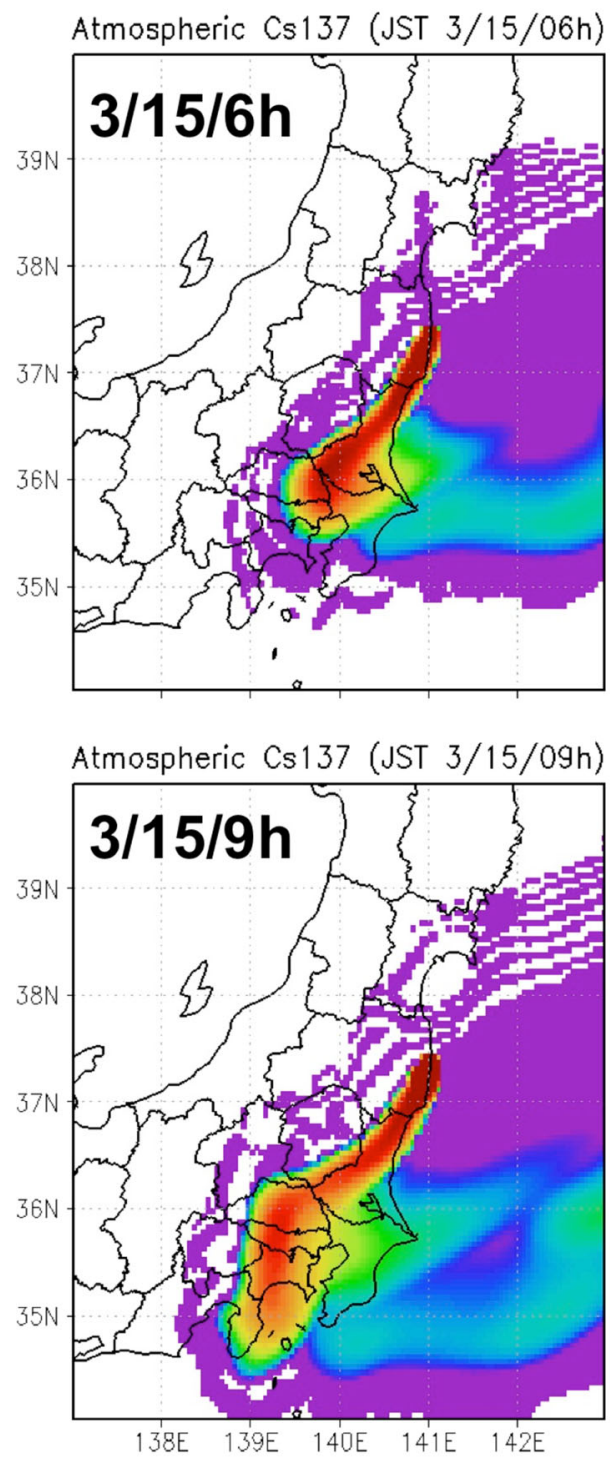

Katata
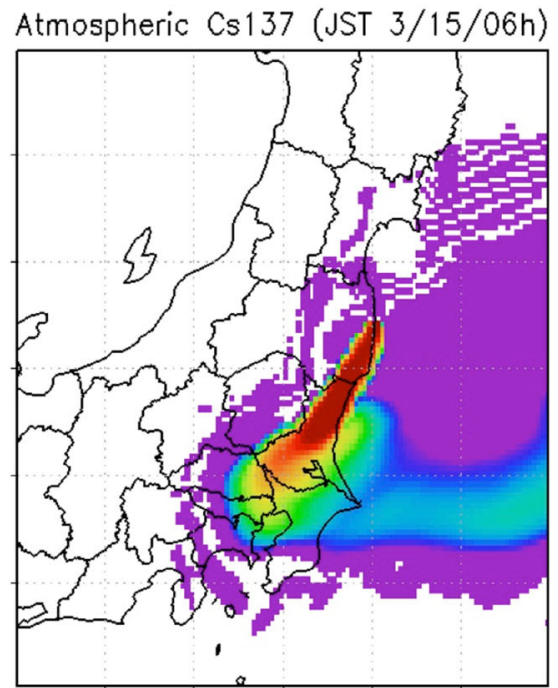

Atmospheric Cs137 (JST 3/15/09h)

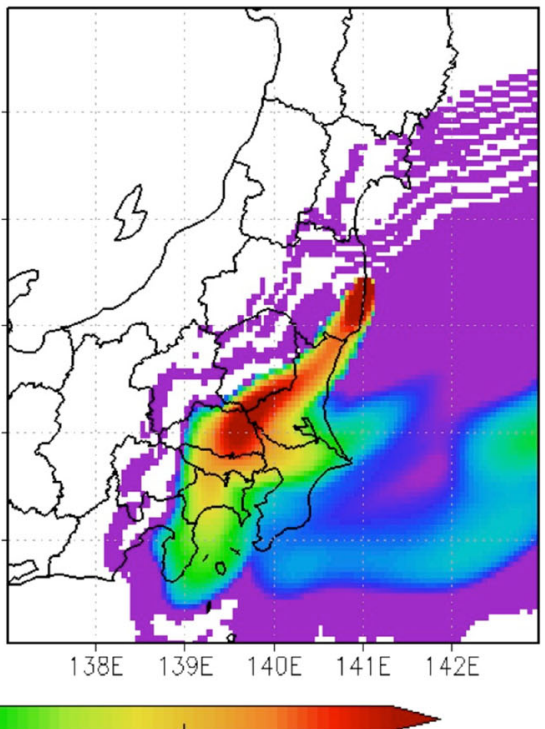

100

\section{Atmospheric concentration $\left({ }^{137} \mathrm{Cs}, \mathrm{Bq} \mathrm{m}^{-3}\right)$}

Fig. 10 Atmospheric ${ }^{137} \mathrm{Cs}$ distributions simulated by the $\mathrm{N}$-model at 3/15/6 h and 3/15/9 $\mathrm{h}$ with emission scenarios by Terada et al. (2012) and Katata et al. (2015)

Fig. 6b, panels 15:00 to 18:00, and Fig. 9e. Tsuruta et al. (2014) also identified this northern branch. However, Figs. $6 \mathrm{~b}$ and $9 \mathrm{e}$ show that without the plumes crossing the Abukuma mountains, the simulated plumes were too narrow to explain the elevated observed concentrations at the SPM sites in the Nakadori region. This problem is probably due to the spatial resolution (3$5 \mathrm{~km}$ ), which is too coarse to simulate the fine-scale orographic horizontal advection of the plume traversing the Abukuma Mountains.

Subsequently, precipitation occurred over the Nakadori and Hamadori regions during the night of 15 March, lasting until the morning of 16 March and dissipating plume P3, as shown in Fig. 6b, panels 7:00 to 9:00, and Fig. 9f. These figures indicate that the next plume, $\mathrm{P} 4$, was transported by a northerly wind that became dominant during 
the morning of March 16, due to increased activity of the low in the Pacific (Fig. 4b). The SPM sites in the Choshi Peninsula observed a high concentration for several hours during the morning of 16 March, although the simulated plume failed to reach the target area (Fig. 9f). A possible explanation for the model failure is that the simulated aerosol height was higher than the actual height. According to the JMA analysis, there was considerable wind shear during the morning of 16 March, i.e., a northeasterly wind prevailed below $600 \mathrm{~m}$, and a northwesterly wind prevailed above $600 \mathrm{~m}$, as indicated by the vertical profiles of the atmosphere at the JMA Tsukuba station $\left(36.06^{\circ} \mathrm{N}\right.$, $140.13^{\circ} \mathrm{E}$ ) shown in Fig. 11. In a trajectory analysis using MANAL data, Miyasaka (Takafumi Miyasaka, University of Tokyo, personal communication, 2015) estimated that the aerosol height for the air mass that reached the Kantou region should be lower than $500 \mathrm{~m}$. However, at the latitude $36.7^{\circ} \mathrm{N}$, the simulated aerosols were distributed up to an altitude of $1500 \mathrm{~m}$. Consequently, the simulated aerosols were transported to the Pacific area once they were uplifted to that level; this is indicated by the fact that the plume is not parallel to the $1000-\mathrm{hPa}$ wind vector. There is a report that NICAM-SPRINTARS tends to overestimate the vertical transport of aerosols (Goto et al. 2015b). Another possible reason is overestimation of the wet deposition by the early morning precipitation along the plume on 16 March; the simulation showed this to be shifted slightly southward and was more than the MANAL objective analysis, as indicated by Fig. 6b, panel 3:00.

Another notable difference between observations and the model is that in plume P2, a medium-level concentration of between 1 and $10 \mathrm{~Bq} \mathrm{~m}^{-3}$ was persistently observed in a wide area of Kantou from the evening of 15 March until the morning of 16 March (Fig. 6b, panels 3/

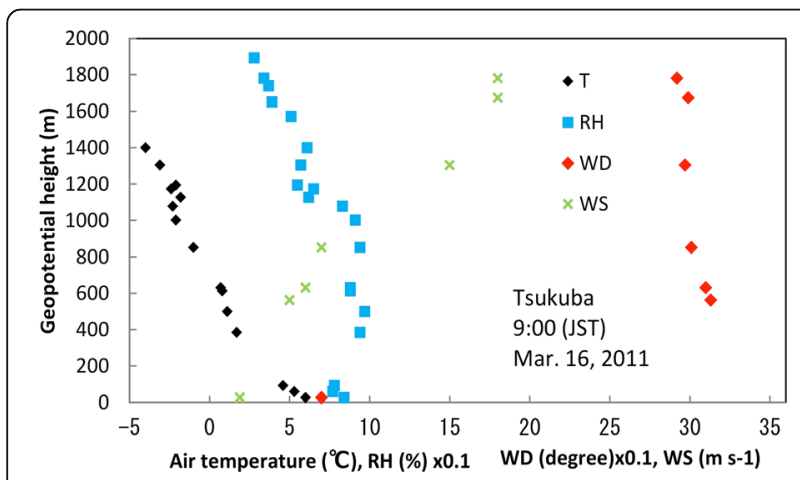

Fig. 11 Vertical profiles of temperature $(T)$, relative humidity $(R H)$, wind direction (WD), and wind speed (WS) versus geopotential height at 3/16/ $9 \mathrm{~h}$ for the JMA Tsukuba station. Wind directions and speeds are at the heights corresponding to the standard isobaric surfaces and characteristic points in the wind profile as determined by the JMA routine measurement protocol
16/0:00 to 7:00; Fig. 9f), whereas the simulation results, in particular, those of the W-model, show a much smaller concentration in the southern part of the Hamadori and Kantou regions. Thus far, it is difficult for us to identify the cause of the model failures for plumes 2 and 4, but it is natural to assume that this is due to the difficulty of accurately simulating transport in a strongly sheared, thin atmosphere.

\section{8-19 March}

Figure 12a, b shows the observed and simulated plumes P5 and P6 in the short-period events of 18 and 19 March. A migrating high-pressure system passed the Japanese islands on those days (Fig. 4c, d), and a weak wind condition was established around the FDNPS. The northwesterly component of the wind field turned southwesterly along the outskirts of the high-pressure zone at approximately 15:00 on 18 March (not shown). The P5 plume driven by the southwesterly wind was subsequently observed at sites north of the FDNPS. It should be noted that the wind vector at 18:00 was relatively perpendicular to the plume with this transport mechanism. A similar oblique relation between the wind vector and the plume axis was also simulated for plume P3 (Fig. 9d) and plumes P7 and P9 (shown below in Fig. 13b, f) under quick changes in the dominant wind direction. These phenomena indicate that the temporal change in wind direction must be accurately reproduced in order to successfully simulate the plume. Moreover, it is also necessary to have higher resolution of the land-sea breeze simulation.

\section{0-21 March}

A subsequent low-pressure system passed the Japanese islands during 20-21 March (Fig. 4e, f). Time sequences of observed and two-model atmospheric ${ }^{137} \mathrm{Cs}$ concentrations with precipitation maps are shown in Fig. 7, and model ensemble results are shown in Fig. 13 for several characteristic times. The figures indicate the dominant westerly wind on 19 March through the early morning of 20 March at the FDNPS blew the plume toward the Pacific, as shown in Fig. 13a (labeled A) and Fig. 7a, panel 6:00; however, during the morning of 20 March, there was an increasing easterly component due to a moving low-pressure system; this blew the plume back westward toward the Hamadori and established plume P7 (labeled B in Fig. 13a), which had a wide distribution covering the Hamadori and its offshore areas, as shown in Fig. 7a, panels 9:00 to 12:00, and Fig. 13a, b. This plume movement is indicated by a thick arrow in Fig. 13a. The simulated distribution of plume P7, however, was very different in the $\mathrm{N}$ - and W-models, resulting in a two-branch pattern of the ensemble mean (A and B in Fig. 13a).

The arrival of P7 at the Choshi Peninsula between 12:00 and 13:00, and its gradual westward spread until $15: 00$, as shown by the rotation of the plume indicated 

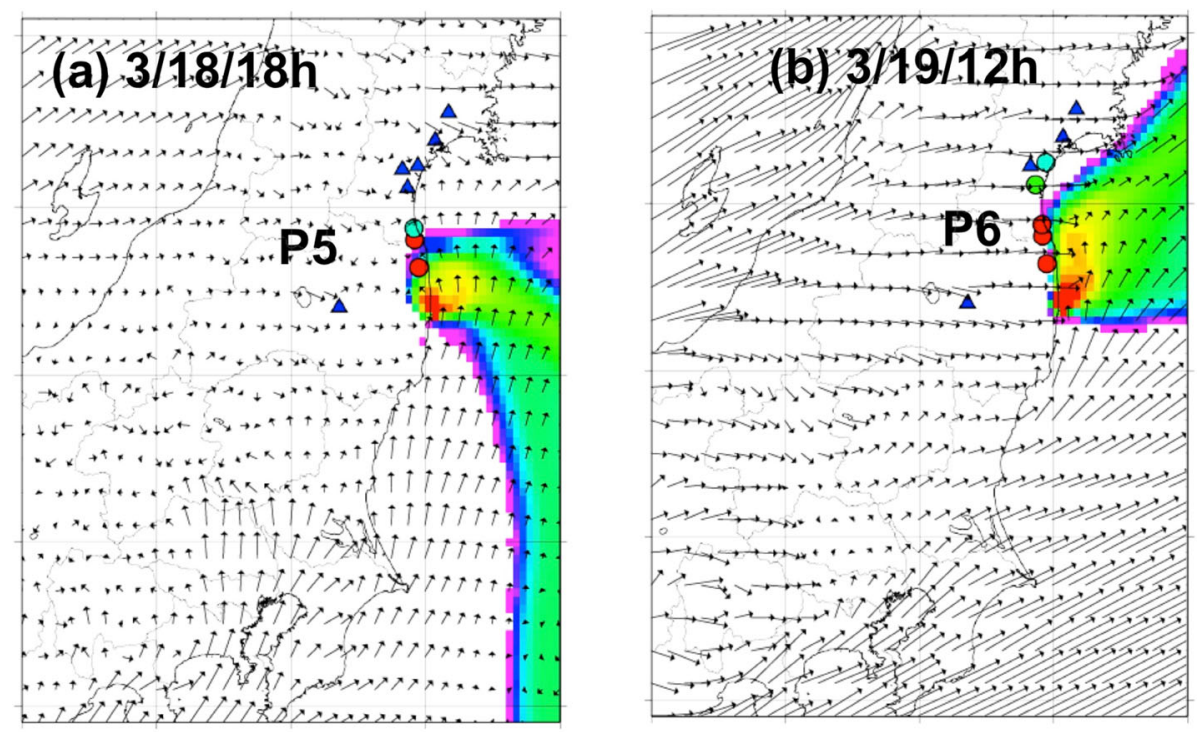

\section{$\begin{array}{lllll}0.01 & 0.1 & 1 & 10 & 100\end{array}$ \\ Atmospheric concentration $\left({ }^{137} \mathrm{Cs}, \mathrm{Bq} \mathrm{m}^{-3}\right)$}

Fig. 12 Maps of observations (colored circles) and model ensemble means of atmospheric ${ }^{137} \mathrm{Cs}$ concentration in a 3/18/18 $\mathrm{h}$ and $\mathbf{b} 3 / 19 / 12 \mathrm{~h}$. Areas identified as plumes with concentration larger than $10 \mathrm{~Bq} \mathrm{~m}^{-3}$ are encircled and labeled with the name of the plume. JMA-MANAL wind vectors at $1000 \mathrm{hPa}$ are superimposed

by a thick arrow in Fig. 13a, was successfully simulated by the N-model, as shown in Fig. 7a, panels 12:00 to 15:00. On the other hand, the W-model failed to simulate this phenomenon. Since precipitation was not involved in the transport process on the morning of 20 March, this failure was considered to be a consequence of more transportation to the upper atmosphere near the emission source, as suggested by the shift to the north (relative to the results of the $\mathrm{N}$-model) of the high concentration around the FDNPS (Fig. 7a, panels 12:00 to 13:00).

Northwestern transport started around 13:00 and lasted until that night, forming plume P8 in the Tohoku region (Fig. 7a, panels 15:00 to 21:00, and Fig. 13b, c). Each model satisfactorily simulates the transportation of plume P8 to the northern part of the Nakadori region, but they are both too coarse to simulate the successive increase in the concentration of ${ }^{137} \mathrm{Cs}$ observed from north to south along the Nakadori channel.

Plumes P7 and P8 started to dissipate at around 3:00 on March 21 (Fig. 7b, panels 1:00 to 7:00, and Fig. 13e). At this time, plume P9 started traveling to Kantou, due to a northeasterly wind ascribed to the combined effect of a migratory system and the Okhotsk low-pressure system (Fig. 4f). Plume P9 reached the Kantou region at around 9:00 on 21 March and collided with the weather front located at the southern part of the Kantou region, as shown by the wind vectors in Fig. 13f. The plume simulated by the N-model had a tongue shape and covered the land area of the northern Chiba prefecture; this was consistent with the distribution of the observed sites characterized by concentrations of more than $100 \mathrm{~Bq} \mathrm{~m}^{-3}$ around the Chiba prefecture and Tokyo Bay, as depicted in Fig. 7b, panels 9:00 to 10:00. However, the $\mathrm{N}$-model plume was too short, and it shifted to the northern area without reaching the observed sites. In this event, the Wmodel also failed to transport ${ }^{137} \mathrm{Cs}$ to the Kantou region. Although not shown by a figure, we found that changing the emission scenario to that of Katata et al. (2015) did not improve the results. Another possibility is that the height of the aerosol layer was overestimated. Figure 14 shows the vertical profile of meteorological parameters at the JMA Tsukuba station at 9:00 on 21 March. The figure indicates that there was a strong temperature inversion at around $300 \mathrm{~m}$ geopotential height. Wind was northeasterly below the inversion and northwesterly above. Therefore, it can be assumed that the modeled aerosol layer was too high, and the aerosols were transported eastward by the northwesterly. This also might be due to the stronger precipitation that was simulated by the models in the Kantou region during the time period 3:00 to 7:00 (Fig. 13b, panels 3:00 to 7:00). These two reasons may 


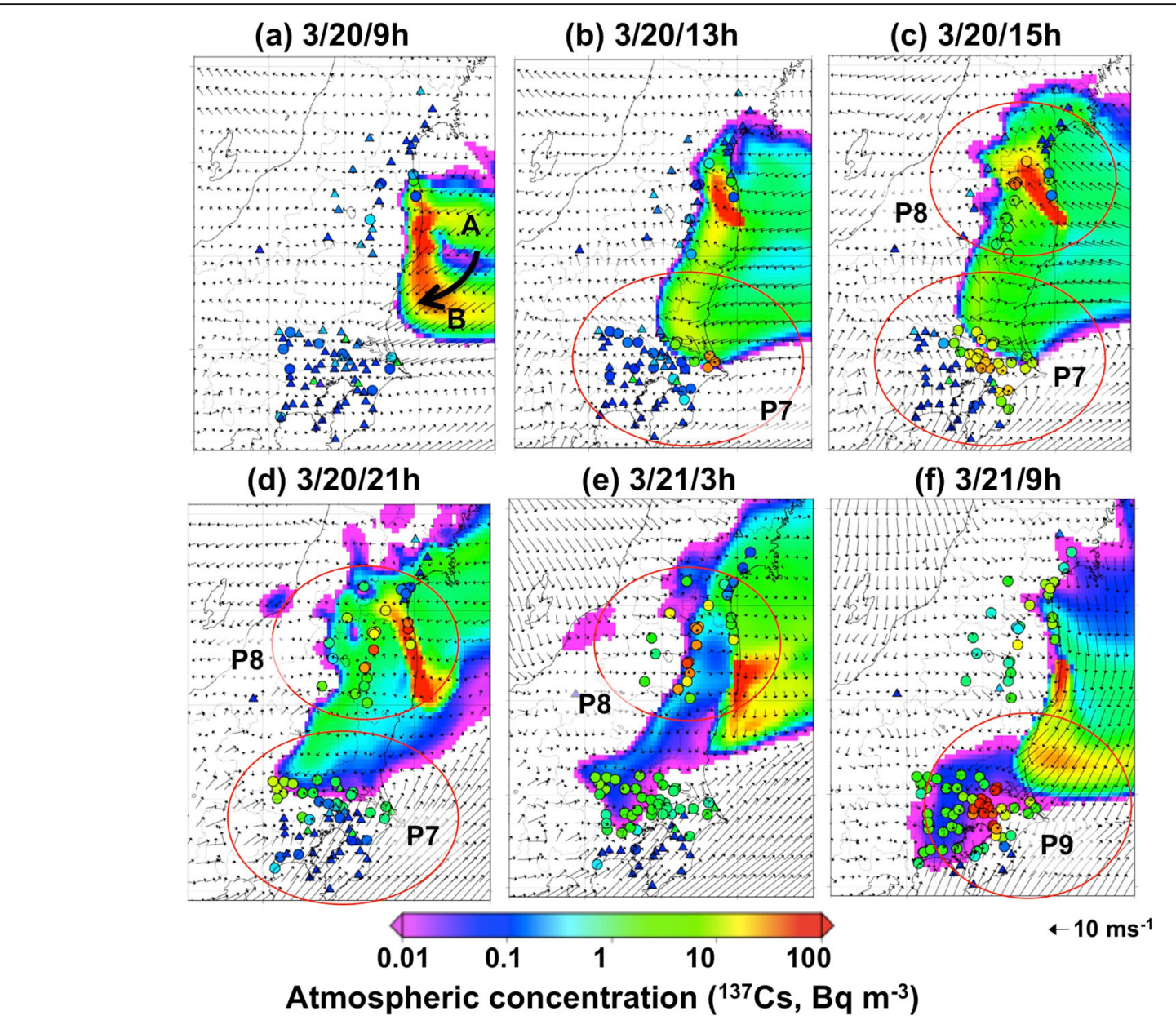

Fig. 13 a-f Maps of observations (colored circles) and model ensemble means of atmospheric ${ }^{137} \mathrm{Cs}$ concentration from 3/20/9 $\mathrm{h}$ to 3/21/9 $\mathrm{h}$. Areas identified as plumes with concentration larger than $10 \mathrm{~Bq} \mathrm{~m}^{-3}$ are encircled and labeled with the name of the plume. JMA-MANAL wind vectors at $1000 \mathrm{hPa}$ are superimposed. Movement of the P7 plume route is indicated by a thick arrow in panel $\mathbf{a}$. See the main text for explanation of labels (a, $\mathbf{b})$

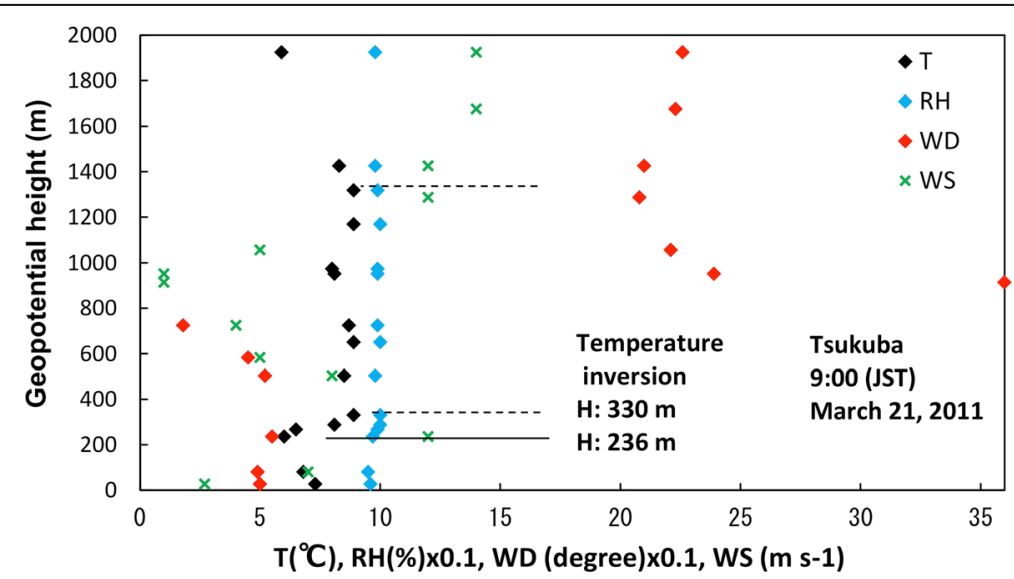

Fig. 14 Vertical profiles of temperature $(T)$, relative humidity $(R H)$, wind direction $(W D)$, and wind speed $(W S)$ versus geopotential height at 3/21/9 $\mathrm{h}$ for the JMA Tsukuba station. Wind directions and speeds are at the heights corresponding to the standard isobaric surfaces and characteristic points in the wind profile as determined by the JMA routine measurement protocol 
explain the quick dissipation of simulated plume P7, in contrast to the observed persistent survival of the plume in the morning of 21 March (Fig. 7b, panels 3:00 to 9:00, and Fig. 13e, f).

\section{Plume routes and time series}

Figure 15 presents a summary of the plume routes analyzed in the preceding sections, and Fig. 16 shows the time series of the observations and the model ensemble mean ${ }^{137} \mathrm{Cs}$ concentrations at several sites. The relevant sites are $\mathrm{B}, \mathrm{C}, \mathrm{E}$, and $\mathrm{J}$ in the Tohoku region and sites 9, 12, and 15 in the Kantou region, as defined by Tsuruta et al. (2014), as well as at the newly added sites K1, K2, and K3.

During a 6-hour period on 15 March, plume P2 spread over a large area of the Kantou region. The coverage was not uniform, and the plume was narrow, as indicated by the peak concentration at sites $9, \mathrm{~K} 1, \mathrm{~K} 2$, and $\mathrm{K} 3$ in Fig. 16b. However, the simulated arrival time was too early, as indicated by the time series and as was already suggested by Fig. 9a.

Plume P3 transported the radioactive material toward the Nakadori region, crossing the Abukuma Mountains, as indicated by the successive peaks over time from the south to the north at sites $\mathrm{E}$ and $\mathrm{C}$ in the afternoon of 15 March (Fig. 16a); the models were too coarse to simulate the detailed progress of the plume along the Nakadori channel. The progress of plumes P2 and P3 is indicated by thick white arrows in Fig. 15.

As shown in Fig. 9f, plume P4 produced a high concentration of ${ }^{137} \mathrm{Cs}$ in the Choshi Peninsula, as shown by the peaks at sites 12 and 15, but the plume did not reach Kantou.

Short-duration plumes P5 and P6 were observed at site J on 18 and 19 March, and they were simulated by the models, but the simulated arrival time was earlier than the observed time. Moreover, the concentration was largely underestimated for the duration of the period.

In the early afternoon of 20 March, plume P7 was transported over a long distance, first to the ocean and then toward land by a clockwise rotation of the plume route, as indicated by the thick white arrow in Fig. 15; it covered a large part of the northern Kantou region, as shown in Fig. 16b.

Plume P8 was transported northward and was redirected southward along the Nakadori region at the northern edge of the Abukuma Mountains. Consequently, the highconcentration area moved from north to south, as illustrated by the time series of observations at sites B, C, and E. However, the models failed to indicate this phenomenon, underestimating the concentration at sites in the Nakadori region, as also shown in Fig. 7a, panels

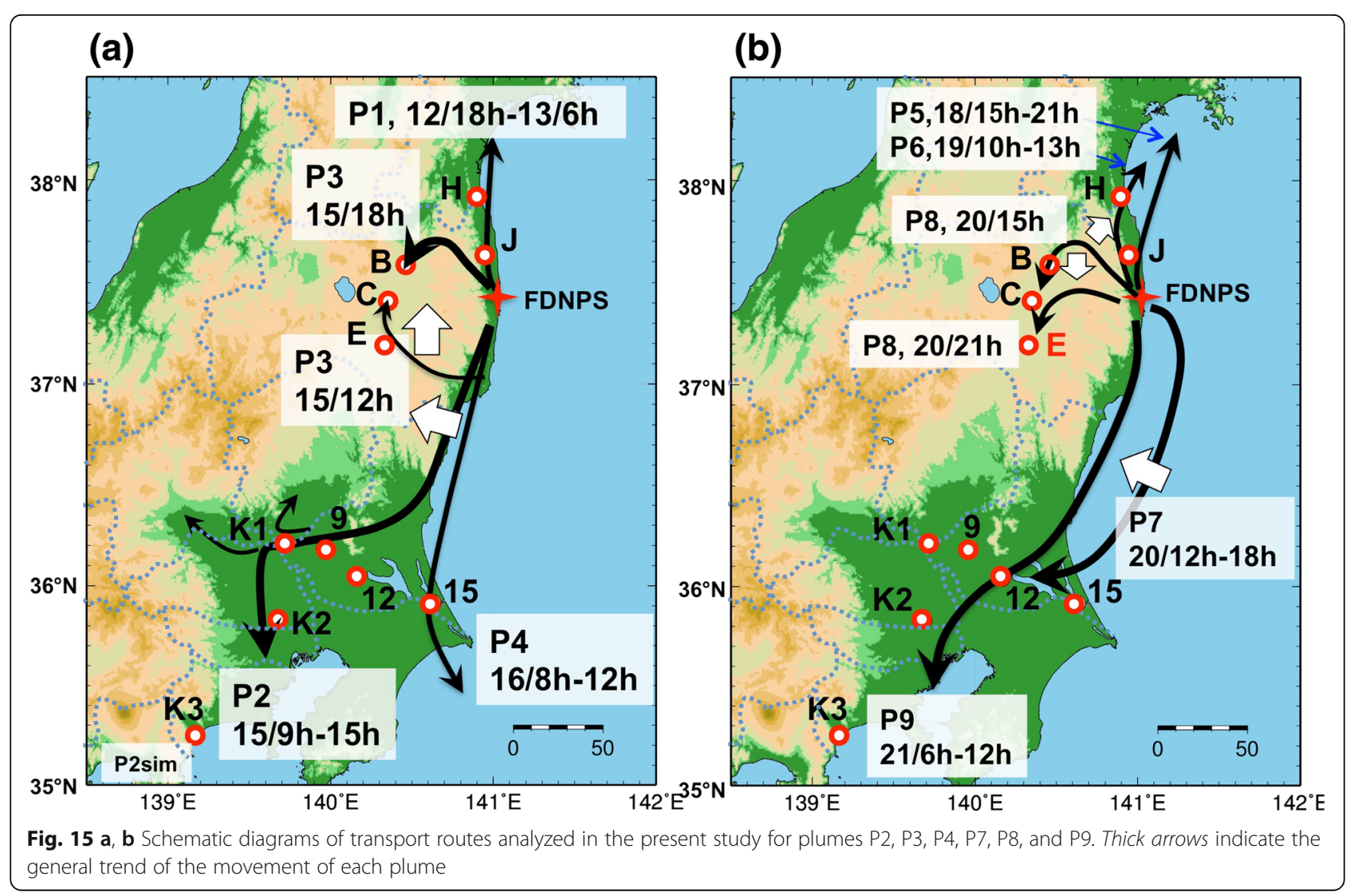




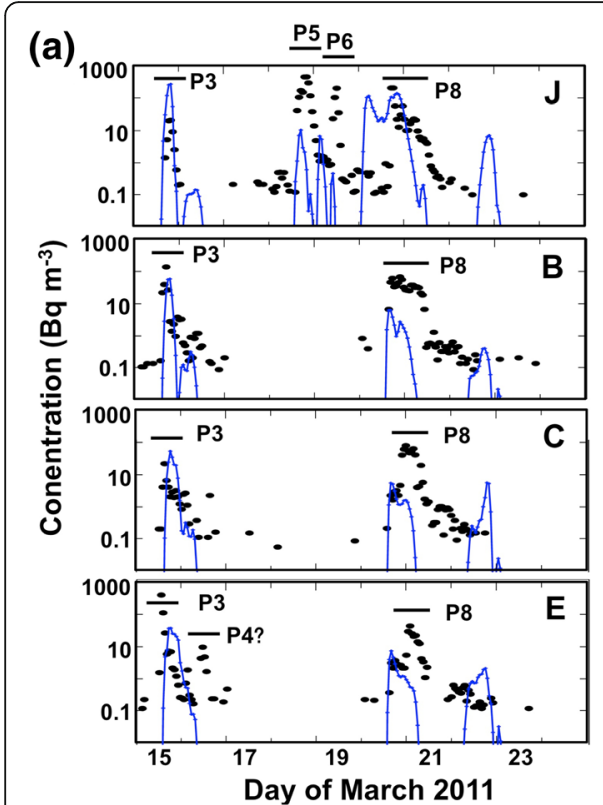

(b)

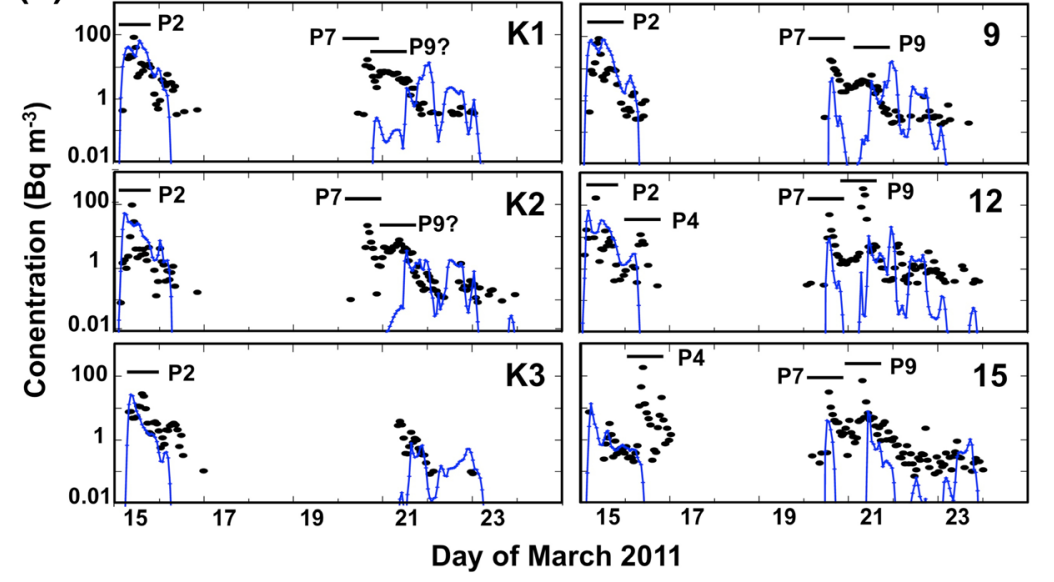

Fig. 16 Time series of observed (dots) and two-model ensemble mean (lines) atmospheric ${ }^{137} \mathrm{Cs}$ concentrations (Bq $\mathrm{m}^{-3}$ ) from 14 to $24 \mathrm{March} \mathbf{a}$ in the Tohoku region at sites J, B, C, and E (see Fig. 15) and $\mathbf{b}$ in the Kantou region at sites 9, 12, 15, K1, K2, and K3 (see Fig. 15)

15:00 to 21:00. At the same time, the wind direction turned from northwest to north at the FDNPS, with the result that the northern part of $\mathrm{P} 8$ covered sites $\mathrm{H}$ and $\mathrm{J}$, as illustrated by the thick northeastward arrow.

Plume P9 began on the morning of 21 March, taking a southern route until it collided with the weather front located in the southern part of the Kantou region. The simulated pattern was too short to correspond with the peaks at sites 9, 12, and 15 at 9:00; however, this is due to the complex transport and precipitation processes discussed in the preceding sections. It is difficult to determine if the peaks at sites $\mathrm{K} 1$ and $\mathrm{K} 2$ on 21 March were caused by a persistent tail from plume P7 or by plume P9, because the observed concentration remained high without a clear separation between plumes, as indicated by the distribution maps shown in Figs. 7b and 13d-f.

\section{Conclusions}

As has been discussed in the preceding sections, we found that a combined analysis of observed and model ensemble data is a useful method for analyzing the development of plumes and the distribution of radioactive materials, but neither approach alone is adequate. Although the SPM observational data are unique and of high density, they are not sufficient to show the detailed distribution structure of the atmospheric ${ }^{137} \mathrm{Cs}$, because the transport mechanism was complex, varied over time, and depended on the local meteorological and geographical conditions. Although in some cases, the models failed to simulate the exact location and time of arrival of the plumes at the SPM sites, the spatial and temporal development of the plume structure was adequately simulated, making it possible to understand how the atmospheric ${ }^{137} \mathrm{Cs}$ was distributed.

The following statement is characteristic of the target area and period, and it is relevant to the atmospheric transportation of radioactive materials: during the analysis period in the spring season in East Asia, migratory pressure systems periodically brought radioactive materials to the Japanese land area, producing somewhat similar plume development patterns. For instance, two peaks can be seen in the time series: one due to plumes P2 and P3 followed by P4, during 15-16 March, and the other due to plumes P7 and P8 followed by P9, during 20-21 March. The first peak (P3 on 15-16 March) was caused by a change in the wind field to northeasterly and later to southeasterly, as a migratory low-pressure system progressed toward the Japanese islands. The second peaks (P4 and P9) were caused by a northerly wind after the low-pressure system had passed from the Japanese islands to the Pacific. When the height of the aerosol layer was overestimated, plumes P4 and P9 deviated eastward due to the westerly wind in the upper layers, such as at $900 \mathrm{hPa}$, as shown in Figs. 9f and 13f.

Future tasks include improving the present method, such as by conducting sensitivity tests for (1) different emission scenarios, for example, those by Katata et al. (2015); (2) plume height based on different model-layering setups; (3) wet deposition processes with different parameterizations; and (4) material transport across and along the northern edge of the Abukuma Mountains. Relevant to task 4, a report by Sekiyama et al. (2015) has claimed 
that there was no significant difference between $3-\mathrm{km}$ and 500-m grid simulations of the JMA nonhydrostatic model for horizontal transport of radioactive material. However, in view of the differences in model parameterization for orographic waves, further analysis of this is necessary. It is also necessary to increase the spread of the model ensemble in order to obtain a more accurate reconstruction of the plume transportation. Another interesting and unresolved problem is the area of mid-level concentration, from 1 to $10 \mathrm{~Bq} \mathrm{~m}^{-3}$, in the Kantou region that persisted from the evening of 20 March to the morning of 21 March. It is also important to carefully evaluate the performance of the model when simulating the vertical stratification of the atmosphere, which controls the dry deposition process. A future study should address aerosol survival under strong but stochastic precipitation conditions. On the other hand, SCJ (2014) suggested that all of the models evaluated tended to underestimate wet deposition in weak precipitation conditions. Consequently, it may be necessary to develop a nonlinear parameterization of the precipitation rate.

We have analyzed only $25 \%$ of the total SPM sampling tapes to date, so additional analysis is necessary. Further efforts are also needed to collect missing observational data, in particular, in the Hamadori region, in order to investigate the detailed atmospheric transportation processes that could not be addressed by the present study. Some of the SPM tapes were discarded by the network before we could retrieve them. Therefore, a special tape conservation effort would be required for large-scale disaster events. We expect the present study to be useful for future research.

\begin{abstract}
Abbreviations
CMAQ: Community multiscale air quality; FDNPS: Fukushima Daiichi Nuclear Power Station; JAEA: Japan Atomic Energy Agency; JAXA: Japan Aerospace eXploration Agency; JMA: Japan Meteorological Agency; JST: Japan standard time; MANAL: Mesoscale objective-analysis data; MEXT: Ministry of Education, Culture, Sports, Science and Technology, Japan; NICAM: Nonhydrostatic icosahedral atmospheric model; NSW6: NICAM single-moment scheme with six water categories; SCJ: Science Council of Japan; SPM: Suspended particulate matter; SPRINTARS: Spectral radiation-transport model for aerosol species; TEPCO: Tokyo Electric Power Company; UNCEAR: United Nations Scientific Committee on the Effects of Atomic Radiation; UTC: Coordinated universal time; WRF: Weather Research and Forecast Model
\end{abstract}

\section{Acknowledgements}

We greatly appreciate the kind support of $\mathrm{H}$. Tomita and H. Yashiro of RIKEN/AICS. We also thank all the local governments who offered the used SPM filter tapes at the request of the Ministry of the Environment, Japan. We have cited the weather maps of Japan Meteorological Agency. We used the meteorological data sets of the Japan Meteorological Agency.

\section{Funding}

Parts of this research were supported by funds from MOE/GOSAT2, JST/ CREST/EMS/TEEDDA, JAXA/EarthCARE\&GCOM-C, MEXT/KAKENHI/Innovative Areas 2409, MOEJ/ERTDF/S-12 and 5-1501, and the National Regulation Agency, Japan.

\section{Authors' contributions}

TN was the principal investigator of the modeling and SPM data analysis projects, the chair of the SCJ Model Comparison Committee, designed the combined analysis, and drafted the manuscript. SM, DG, JU, TT, and MS conducted the numerical simulations with NICAM-SPRINTARS. YM and TO conducted the numerical simulations with WRF-CMAQ. HT, TO, and ME conducted the SPM tape analysis. All co-authors participated in discussions about the results and commented on the original manuscript. All authors read and approved the final manuscript.

\section{Authors' information}

Correspondence and requests for materials should be addressed to TN (terry-nkj@nifty.com).

\section{Competing interests}

The authors declare that they have no competing interests.

\begin{abstract}
Author details
${ }^{1}$ Atmosphere and Ocean Research Institute (AORI), The University of Tokyo, 5-1-5 Kashiwanoha, Kashiwa, Chiba 277-8568, Japan. ${ }^{2}$ Earth Observation Research Center (EORC), Japan Aerospace Exploration Agency (JAXA), 2-1-1 Sengen, Tsukuba, Ibaraki 305-8505, Japan. ${ }^{3}$ National Institute for Environmental Studies (NIES), 16-2 Onogawa, Tsukuba, Ibaraki 305-8506, Japan. ${ }^{4}$ Remote Sensing Technology Center of Japan, 3-17-1 Toranomon, Minato-ku, Tokyo 105-0001, Japan. ${ }^{5}$ Research Institute for Applied Mechanics, Kyushu University, Kasuga Park 6-1, Kasuga, Fukuoka 816-8580, Japan.

${ }^{6}$ Graduate School of Science and Engineering, Tokyo Metropolitan University, 1-1 Minami-Osawa, Hachioji, Tokyo 192-0397, Japan.
\end{abstract}

Received: 10 June 2016 Accepted: 5 January 2017

Published online: 23 January 2017

\section{References}

Byun DW, Ching JKS (1999) Science algorithms of the EPA Models-3 Community Multi-Scale Air Quality (CMAQ) modeling system. Rep. USA EPA/600/R-99/ 030. US Environmental Protection Agency, Washington

Byun D, Schere KL (2006) Review of the governing equations, computational algorithms, and other components of the Models-3 Community Multiscale Air Quality (CMAQ) modeling system. Appl Mech Rev 59:51-77

Dai T, Goto D, Schutgens NAJ, Dong X, Shi G, Nakajima T (2014) Simulated aerosol key optical properties over global scale using an aerosol transport model coupled with a new type of dynamic core. Atmos Environ 82:71-82. doi:10.1016/j.atmosenv.2013.10.018

Goto D, Dai T, Satoh M, Tomita H, Uchida J, Misawa S, Inoue T, Tsuruta H, Ueda K, Ng CFS, Takami A, Sugimoto N, Shimizu A, Ohara T, Nakajima T (2015a) Application of a global nonhydrostatic model with a stretched-grid system to regional aerosol simulations around Japan. Geosci Model Dev 8:235-259. do::10.5194/gmd-8-235-2015

Goto D, Nakajima T, Dai T, Takemura T, Kajino M, Matsui H, Takami A, Hatakeyama S, Sugimoto N, Shimizu A, Ohara T (2015b) An evaluation of simulated particulate sulfate over East Asia through global model intercomparison. J Geophys Res Atmos 120(12):6247-6270. doi:10.1002/ 2014JD021693

JAEA (2012) JAEA Open Workshop 'Reconstruction of the Emission and diffusion processes of materials released by the Fukushima Daiichi Atomic Power Plant accident', March 6, 2012, Tokyo, http://nsec.jaea.go.jp/ers/environment/envs/ FukushimaWS/index.htm. Accessed 18 Jan 2017

Kaneyasu N, Ohashi H, Suzuki F, Okuda T, Ikemori F (2012) Sulfate aerosol as a potential transport medium of radiocesium from the Fukushima nuclear accident. Environ Sci Technol 46:5720-5726. doi:10.1021/es204667h

Katata G, Chino M, Kobayashi T, Terada H, Ota M, Nagai H, Kajino M, Draxler R, Hort MC, Malo A, Torii T, Sanada Y (2015) Detailed source term estimation of the atmospheric release for the Fukushima Daiichi Nuclear Power Station accident by coupling simulations of an atmospheric dispersion model with an improved deposition scheme and oceanic dispersion model. Atmos Chem Phys 15:1029-1070. doi:10.5194/acp-15-1029-2015

Lin YL, Farley RD, Orville HD (1983) Bulk parameterization of the snow field in a cloud model. J Clim Appl Meteorol 22:1065-1092

Mellor GL, Yamada T (1982) Development of a turbulence closure model for geophysical fluid problems. Rev Geophys Space Phys 20:851-875 
Mészáros R, Leelőssy Á, Kovács T, Lagzi I (2016) Predictability of the dispersion of Fukushima-derived radionuclides and their homogenization in the atmosphere. Sci Rep 6:19915. doi:10.1038/srep19915

MEXT (2011) Airborne monitoring results after the first one conducted by the Ministry of Education, Culture, Sports, Science, and Technology in April 2011 are summarized by the Nuclear Regulation Authority. http://radioactivity.nsr. go.jp/ja/list/362/list-1.html. Accessed 6 May 2011

Morino Y, Ohara T, Nishizawa M (2011) Atmospheric behavior, deposition, and budget of radioactive materials from the Fukushima Daiichi Nuclear Power Plant in March 2011. Geophys Res Lett 38:L00G11. doi:10.1029/2011GL048689

Morino Y, Ohara T, Watanabe M, Hayashi S, Nishizawa M (2013) Episode analysis of deposition of radiocesium from the Fukushima Daiichi Nuclear Power Plant accident. Environ Sci Technol 47:2314-2322. doi:10.1021/es304620x

Nakajima T, Ohara T, Uematsu M, Onda Y (eds) (2014) Radioactive environmental pollution from the Fukushima Daiichi Nuclear Power Station accident: earth science perspectives. University of Tokyo Press, Tokyo (in Japanese)

Nakanishi M, Niino H (2004) An improved Mellor-Yamada level 3 model with condensation physics: its design and verification. Bound Layer Meteorol 112:1-31

Oura Y, Ebihara M, Tsuruta H, Nakajima T, Ohara T, Ishimoto M, Sawahata H, Katsumura Y, Nitta W (2015) A database of hourly atmospheric concentrations of radiocesium $\left({ }^{134} \mathrm{Cs}\right.$ and $\left.{ }^{137} \mathrm{Cs}\right)$ in suspended particulate matter collected in March 2011 at 99 air pollution monitoring stations in eastern Japan. J Nucl Radiochem Sci 15(2):1-12

Satoh M, Matsuno T, Tomita H, Miura H, Nasuno T, Iga S (2008) Nonhydrostatic Icosahedral Atmospheric Model (NICAM) for global cloud resolving simulations. J Comput Phys 227:3486-3514

SCJ (2014) A review of the model comparison of transportation and deposition of radioactive materials released to the environment as a result of the Tokyo Electric Power Company's Fukushima Daiichi Nuclear Power Plant accident, Report of Committee on Comprehensive Synthetic Engineering, Science Council of Japan, http://www.jpgu.org/scj/report/20140902scj_report_e.pdf. Accessed 18 Jan 2014

Sekiyama TT, Kunii M, Kajino M, Shimbori T (2015) Horizontal resolution dependence of atmospheric simulations of the Fukushima nuclear accident using 15-km, 3-km, and 500-m grid models. J Meteorol Soc Jpn 93:49-64

Skamarock WC, Klemp JB, Dudhia J, Gill DO, Barker DM, Duda MG, Huang XY, Wang W, Powers JG (2008) A description of the Advanced Research WRF Version 3. NCAR/TN.475 + STR. National Center for Atmospheric Research, Boulder

Sparmacher H, Fulber K, Bonka H (1993) Below-cloud scavenging of aerosol particles: particle-bound radionuclides-experimental. Atmos Environ 27(4): 605-618. doi:10.1016/0960-1686(93)90218-N

Sportisse B (2007) A review of parameterizations for modelling dry deposition and scavenging of radionuclides. Atmos Environ 41:2683-2698. doi:10.1016/j. atmosenv.2006.11.057

Stohl A, Seibert P, Wotawa G, Arnold D, Burkhart JF, Eckhardt S, Tapia C, Vargas A, Yasunari TJ (2012) Xenon-133 and cesium-137 releases into the atmosphere from the Fukushima Dai-ichi nuclear power plant: determination of the source term, atmospheric dispersion, and deposition. Atmos Chem Phys 12: 2313-2343. doi:10.5194/acp-12-2313-2012

Takemura T, Okamoto H, Maruyama Y, Numaguti A, Higurashi A, Nakajima T (2000) Global three-dimensional simulation of aerosol optical thickness distribution of various origins. J Geophys Res 105:17853-17873

Takemura T, Nakamura H, Takigawa M, Kondo H, Satomura T, Miyasaka T, Nakajima T (2011) A numerical simulation of global transport of atmospheric particles emitted from the Fukushima Daiichi Nuclear Power Plant. SOLA 7: 101-104. doi:10.2151/sola.2011-026

Terada H, Katata G, Chino M, Nagai H (2012) Atmospheric discharge and dispersion of radionuclides during the Fukushima Dai-ichi Nuclear Power Plant accident, part II: verification of the source term and analysis of regional-scale atmospheric dispersion. J Environ Radioact 112:141-154

Tomita H, Satoh M (2004) A new dynamical framework of nonhydrostatic global model using the icosahedral grid. Fluid Dyn Res 34:357-400

Tsuruta H, Oura Y, Ebihara M, Ohara T, Nakajima T (2014) First retrieval of hourly atmospheric radionuclides just after the Fukushima accident by analyzing filter-tapes of operational air pollution monitoring stations. Sci Rep 4:6717. doi:10.1038/srep06717

Uchida J, Mori M, Suzuki K, Satoh M, Nakamura H, Nakajima T (2015) Error and energy budget analysis of a non-hydrostatic stretched-grid global circulation model. Mon Weather Rev 144:1423-1447. doi:10.1175/MWR-D-15-0271.1
Wang W, Bruyère C, Duda M, Dudhia J, Gill D, Lin H-C, Michalakes J, Rizvi S, Zhang X, Beezley JD, Coen JL, Mandel J (2012) User's guide: Advanced Research WRF (ARW) Version 3.3 modeling system. http://www.mmm.ucar. edu/wrf/users/. Accessed 12 June 2016

Yasunari TJ, Stohl A, Hayano RS, Burkhart JF, Eckhardt S, Yasunari T (2011) Cesium-137 deposition and contamination of Japanese soils due to the Fukushima nuclear accident. PNAS 108:19530-19534. doi:10.1073/pnas.1112058108

Yumimoto K, Morino Y, Ohara T, Oura Y, Ebihara M, Tsuruta H, Nakajima T (2016) Inverse modeling of the 137Cs source term of the Fukushima Dai-ichi Nuclear Power Plant accident constrained by a deposition map monitored by aircraft. J Environ Radioact 164:1-12

\section{Submit your manuscript to a SpringerOpen ${ }^{\circ}$ journal and benefit from:}

- Convenient online submission

- Rigorous peer review

- Immediate publication on acceptance

- Open access: articles freely available online

- High visibility within the field

- Retaining the copyright to your article

Submit your next manuscript at $>$ springeropen.com 